Full Paper

\title{
Arbeitszeitbedarf bei der Futterproduktion in Südtirol
}

\author{
Labour requirements for the forage production in South Tyrol \\ Fabbisogno di manodopera per la produzione di foraggio in Alto Adige
}

Giovanni Peratoner ${ }^{1}$, Ulrich Figl ${ }^{1}$, Claudia Floriann ${ }^{1}$, Franziska Mairhofer ${ }^{1}$

${ }^{1}$ Versuchszentrum Laimburg, Pfatten, Italien

\section{ABSTRACT}

Forage production in mountain areas is an important production factor for livestock farms as well as a source of ecosystem services for the society. The labour requirements, however, are sometimes considerable. The reference values from other countries in the Alpine region are subject to different operational and topographical conditions. The aim of this study is therefore to generate reliable, local and specific guideline values of the labour requirements for the forage production in mountain areas. 20 farms in the Puster Valley were selected and 100 to 109 plots per year, assigned to the categories meadow, grassland use combining mowing and grazing and maize grown for silage were investigated. The data collection took place from 2011 to 2013. The farmers systematically recorded the working times including set-up and dismantling times as well as travel times for each single field operation. The slope showed a highly significant effect on the total labour requirement for meadows. This can be explained in particular by the strong increase in manual labour for forage harvesting. The labour requirement for harvesting was five times higher for the highest slope class than for the lowest. The altitude, on the contrary, had no significant effect. Also for grassland use combining mowing and grazing only the slope affected the total labour requirement. For maize grown for silage, harvesting and ensiling were the most time-consuming categories, followed by soil tillage. Taking into account the local conditions, the methodology used and its constraints, the results provide useful reference values of the labour requirements in the agricultural practice in South Tyrol.

\section{KEYWORDS}

mountain grassland, meadows, labour input, topography, reference values

\section{CITE ARTICLE AS}

Peratoner Giovanni, Figl Ulrich, Florian Claudia et al. (2021). Labour requirements for the forage production in South Tyrol. Laimburg Journal 03/2021 DOI: $10.23796 / \mathrm{LJ} / 2021.008$

\section{CORRESPONDING AUTHOR}

Giovanni Peratoner Laimburg 6, Pfatten, I-39040 Auer (BZ), Italien giovanni.peratoner@laimburg.it +390471969661 


\section{EINLEITUNG}

Der Bergfutterbau ist ein wichtiger Produktions- und Beschäftigungsfaktor für die lokale Bevölkerung im Bereich der Tierhaltung. In der Autonomen Provinz Bozen entfallen etwa drei Viertel der Futterproduktion auf Wiesen in ihren unterschiedlichen Bewirtschaftungsintensitäten [1]. Die Futterproduktion, insbesondere in ihren extensiven Formen, stellt für die Gesellschaft eine wichtige Quelle von Ökosystemdienstleistungen dar. Sie reichen von landschaftlichen Aspekten über pflanzliche Biodiversität bis hin zum Schutz vor Bodenerosion [2]. Der Erhalt von Futterflächen kann am besten durch eine standortgerechte landwirtschaftliche Nutzung gewährleistet werden, jedoch sind derzeit weniger topografisch und klimatisch günstig gelegene Gebiete aufgrund der laufenden sozioökonomischen Veränderungen von einer Aufgabe bedroht. Andererseits sind gerade die Grünlandflächen mit schlechter Zugänglichkeit und Einschränkungen bei der Mechanisierung und der Intensivierung diejenigen, die die größte Vielfalt an Pflanzenarten aufweisen [3]. Maßnahmen zur Unterstützung der Futtermittelwirtschaft in Berggebieten wie z.B. ländliche Entwicklungspläne, und die Quantifizierung dieser Maßnahmen dürfen die Verfügbarkeit zuverlässiger und objektiver Daten über die Futtermittelproduktionskosten nicht außer Acht lassen. Dies gewährleistet eine zielgerichtete und effiziente Zuweisung der öffentlichen Ressourcen. Im Alpenraum gibt es mehrere bibliografisch dokumentierte Studien zur Quantifizierung der Arbeitszeit für die Futterproduktion in den Bergen (siehe
z.B. [4] [5]). Da diese Ergebnisse jedoch mit den sozioökonomischen Bedingungen und der Agrarstruktur der Länder, in denen die Daten erhoben wurden (Österreich und Schweiz in den oben genannten Fällen), zusammenhängen, besteht ein Bedarf an Informationen über das lokale Gebiet in der Provinz Bozen. Aus diesem Grund hat das Versuchszentrum Laimburg in Zusammenarbeit mit der Abteilung Landwirtschaft der Autonomen Provinz Bozen, der EdmundMach-Stiftung und der ehemaligen Bergbauernberatung das Projekt BLW-gW-11-1 (Quantifizierung der Kosten für die Produktion von Futtermitteln in der Provinz Bozen) von 2011-2014 durchgeführt [6] [7] [8]. Im Rahmen des Projektes BLW-gw-18-3 (Erarbeitung von Richtwerten für die Arbeitszeiten in der Südtiroler Futtermittelproduktion) wurde eine zusätzliche gezielte Auswertung der Daten des Projektes BLW-gw11-1 mit Schwerpunkt auf den Arbeitszeitbedarf für die Futterproduktion auf Anregung eines externen Projektvorschlags des Südtiroler Bauernbundes durchgeführt.

In der Futterproduktion sind die Arbeitszeiten erheblich und es wird erwartet, dass sie von den topografischen Eigenschaften und vor allem von der Hangneigung beeinflusst werden. Die Südtiroler Grünlandwirtschaft ist zum Teil von steilen Hängen geprägt: im Jahr 2008 wies etwas mehr als ein Drittel der Gesamtfläche (36\%) eine leichte Hangneigung unter $20 \%$ auf, etwas weniger als die Hälfte der Fläche (45\%) hatte eine mittlere Ausprägung (21-50\% Hangneigung) und knapp $20 \%$ der Fläche war steil (51-60\% Hangneigung) oder sehr steil (> 60\% Hang- neigung) [9]. Bis dato sind keine lokalen, spezifischen Richtwerte für den Arbeitszeitbedarf bei der Futterproduktion verfügbar. Zwar liegen Richtwerte aus anderen Ländern im Alpenraum (Österreich, Schweiz, Deutschland) vor [4] [5] [10] [11] [12] [13] [14] [15], aber aufgrund der abweichenden betrieblichen (Betriebsgröße, Mechanisierungsgrad) und topografischen Gegebenheiten (vor allem der Hangneigung) sowie der Berücksichtigung verschiedener Kombinationen von Einflussgrößen (Tab. 1) ist die Repräsentativität dieser Ergebnisse zumindest lokal zu überprüfen.

Das Ziel dieser Studie ist daher, zuverlässige, lokale Richtwerte hinsichtlich der Arbeitszeiten für die Futterproduktion im Berggebiet zu erarbeiten. Sie werden somit den Beratungseinrichtungen für Berechnungen (z.B. Finanzierungspläne) und Argumentationen (z.B. Betriebszweigvergleiche, Investitionsplanungen) zur Verfügung stehen.

\section{MATERIAL UND METHODEN}

\section{DATENERHEBUNG}

Der Untersuchungsplan wurde ursprünglich mit dem Ziel erstellt, den Effekt von Neigung und Meereshöhe der Futterflächen auf die Kosten der Futterproduktion zu untersuchen [8]. In Zusammenarbeit mit der Abteilung Landwirtschaft wurden für jeden Bezirk Betriebe gesucht, die repräsentative Grünlandflächen mit jeder Kombination von Meereshöhe und Neigung besaßen und deren Betriebsleiter bereit waren, die eigenen Arbeitszeiten für die Futterproduktion über

Tab. 1: Berücksichtigte Einflussgrößen bei der Schätzung des Arbeitszeitbedarfs und Einfluss des jeweiligen Faktors nach verschiedenen Literatur quellen. $\uparrow=$ Zunahme des Arbeitszeitbedarfs bei zunehmendem Wert der Einflussgröße; $\downarrow=$ Abnahme des Arbeitszeitbedarfs bei zunehmendem Wert der Einflussgröße; - = nicht berücksichtigt; GVE = Milchvieh-Großvieheinheiten; FKV = Futterkonservierungsverfahren. *Schätzung auf Betriebsebene, Gesamt-AKh pro gehaltene GVE, **Schätzung auf Parzellenebene, AKh pro ha nur für bestimmte Arbeiten, ***Schätzung auf Parzellenebene, Gesamt-AKh pro ha // Factors considered in estimating the labour requirements and effect of each factor according to various literature sources. $\uparrow=$ increase in labour requirement with increasing value of the influencing variable; $\downarrow=$ decrease in labour requirements with increasing value of the influencing variable; - = not considered; GVE = dairy cattle livestock units; FKV = forage conservation method. *Estimation at farm level, labour requirements in hours per livestock unit kept, **Estimation at field level, labour requirements in hours per hectare only for certain work categories, *** Estimation at field level, labour requirements in hours per hectare.

\begin{tabular}{|c|c|c|c|c|c|}
\hline Literaturquelle & Hangneigung & Schlagfläche & Meereshöhe & Schnitthäufigkeit & $\begin{array}{c}\text { Andere berücksichtigte } \\
\text { Einflussgrößen }\end{array}$ \\
\hline Source & Slope & Field size & Altitude & Cut frequency & Other factors taken into account \\
\hline
\end{tabular}

\begin{tabular}{lccccc} 
Schick 1995* & $\uparrow$ & $\downarrow$ & $\uparrow$ & - & GVE \\
\hline Schick 1995** & $\uparrow$ & $\downarrow$ & - & - & - \\
\hline Greimel et al. 2002*** & $\uparrow$ & - & - & $\uparrow$ & FKV \\
\hline Dilger \& Faulhaber 2006*** & - & - & - & $\uparrow$ & FKV \\
\hline BMLFUW 2008*** & $\uparrow$ & - & - & $\uparrow$ & FKV
\end{tabular}




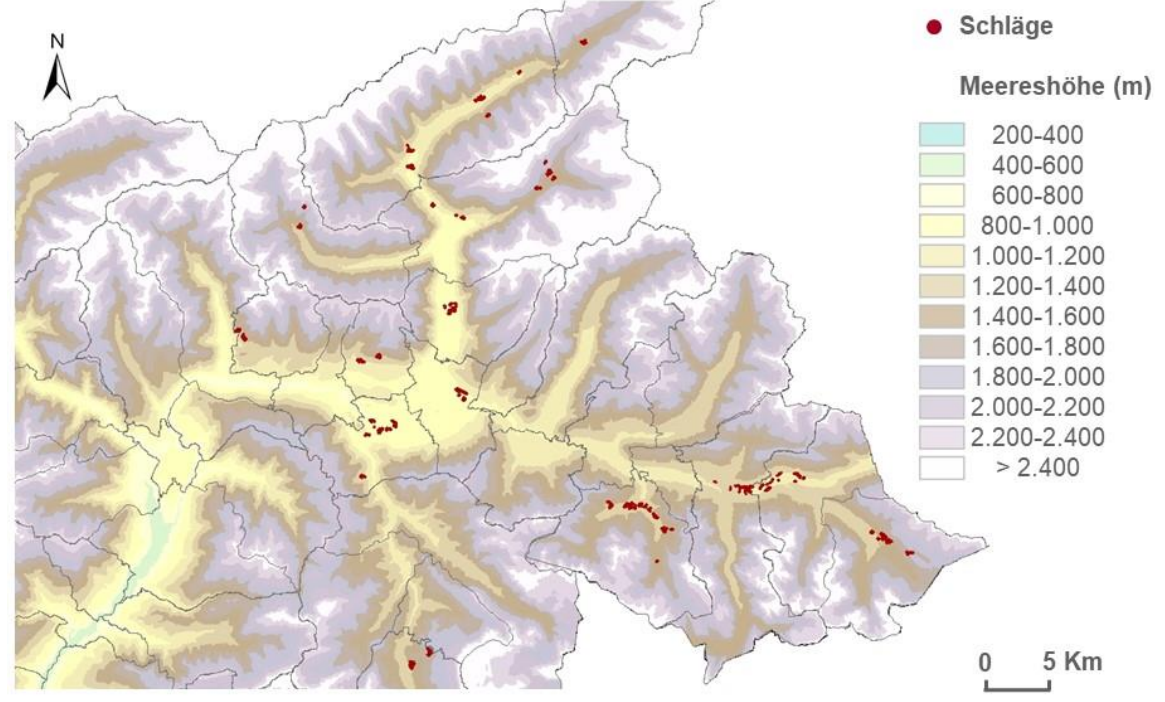

Abb. 1: Lage der untersuchten Schläge im Pustertal // Location of the investigated fields in Pustervalley.

die gesamte Projektdauer (2011-2013) zu erheben. Um den Aufwand für die Betreuung der Datenerhebung möglichst gering zu halten, wurden aus logistischen Gründen 20 Betriebe in 14 Gemeinden im Raum Pustertal ausgewählt (Abb. 1). Als Schläge wurden Flächen definiert, die zu einem einzigen Betrieb gehören, meistens (aber nicht immer) arrondiert sind und vom Betriebsleiter als eigenständige Einheit homogen bewirtschaftet werden.

Die untersuchten Schläge (100 im Jahr 2011,

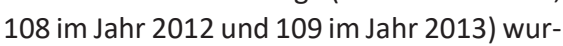
den den Kategorien Wiese, Mähweide und Silomais (letztere waren $9 \mathrm{im}$ Jahr 2011, 10 im Jahr 2012 und 9 im Jahr 2013) zugeteilt. Als Mähweiden wurden Futterflächen definiert, bei denen die Weidenutzung überwog und die höchstens eine Mahd pro Jahr aufwiesen. In diese Kategorie fallen deshalb keine Wiesen hinein, bei denen nur der letzte Aufwuchs beweidet wird. Letztere wurden der Kategorie Wiese zugeordnet.
Die Schläge wurden von den Landwirten auf Papier (Orthofoto in Kombination mit den Katastermappen) eingezeichnet und danach mit ArcGis 10.0 digitalisiert. Mit dem Tool Zonal Statistics as Table wurden die mittlere Schlaggröße, Meereshöhe und Hangneigung jeder Fläche berechnet, basierend auf dem digitalen Geländemodell der Autonomen Provinz Bozen mit einer Auflösung von $20 \mathrm{~m}$. Die erhobenen Schläge sind zwischen der montanen und alpinen Zone verteilt (zwischen 800 und $2100 \mathrm{~m}$ ) und haben eine Hangneigung von $3 \%-86 \%$. Ihre Flächengröße schwankt etwa zwischen 0,1 und 8 ha, mit einer Durchschnittsgröße von einem halben Hektar (Tab. 2). Die Silomaisflächen weisen, was die topografischen Eigenschaften betrifft, weniger Unterschiede auf und haben eine durchschnittliche Hangneigung von $15 \%$ und eine Meereshöhe von 800-950 m. Die Schlaggröße hingegen weist eine starke Variabilität auf (zwischen 0,2 und 1,5 ha).
Zur Berechnung der Arbeitszeiten wurden von den teilnehmenden Landwirten Daten zu den Maschinen/Geräten, Personal und Arbeitsgängen erhoben (Abb. 2)

Die von den Landwirten erfassten Arbeitszeiten, die ausschlaggebend für die vorliegende Auswertung sind, schließen die Zeit zwischen der Ankunft auf dem Feld bis zum Ende des ausgeführten Arbeitsgangs auf dem Feld ein. Um jedoch die Beschreibung der gesamten Arbeitszeit des Personals zu vervollständigen, sind auch die Auf- und Abrüstzeiten und die Anfahrtszeiten von der Hofstelle bis zum Grundstück sowie die Rückfahrzeiten einkalkuliert worden. Für jede Kombination von Maschine und Schlag wurde eine Anfahrtszeit vom Landwirt angegeben. Als Standardanfahrtszeit zur Quantifizierung der zeitlichen Entfernung des Schlags von der Hofstelle wurde die Fahrtzeit mit einem Standardfahrzeug (Mittelwert der Fahrtzeiten mit Transporter und Traktor) herangezogen. Diese Anfahrtszeit wurde auch zur Berechnung der Arbeitszeit jenes Personals herangezogen, das die Grundstücke mit anderen Fahrzeugen erreichte, welche nicht die Maschinen zur Ausübung des jeweiligen Arbeitsgangs waren. Vor Beginn der Erhebung der Arbeitszeiten wurden die Betriebsleiter befragt, die Aufund Abrüstzeiten aller im Betrieb eingesetzten Maschinen und Geräte zu definieren. Ein Fragebogen für die Erfassung der Arbeitszeiten mit einem betriebsspezifischen Verzeichnis des Personals und der Maschinen/Geräte wurde erstellt. Die Datenerhebung wurde während der Saison von einer Technikerin/einem Techniker des Versuchszentrums Laimburg bzw. Mitarbeitern der Abteilung Landwirtschaft sowie der ehemaligen Dienststelle für die Bergbauernberatung begleitet und betreut. An sie/ihn konnten sich die Betriebsleiter zu jeder Zeit wenden, um Informationen über die Methodik

Tab. 2: Anzahl der Betriebe, der Schläge und der erhobenen Arbeitsgänge sowie topografische Eigenschaften der untersuchten Schläge in den Kategorien Wiesen und Mähweiden ( $\mathrm{n}=$ Anzahl, $\min =$ Minimum, $\mathrm{m}=$ Mittelwert, max = Maximum) // Number of farms, fields and operations, as well as topographical characteristics of the fields surveyed in the categories meadows and combination of mowing and grazing $(n=n u m b e r$, min $=m i n i m u m$, $m=$ average, $\max =$ maximum).

\begin{tabular}{|c|c|c|c|c|c|c|c|c|c|c|c|c|}
\hline \multirow[t]{2}{*}{$\begin{array}{l}\text { Jahr } \\
\text { Year }\end{array}$} & \multirow{2}{*}{$\begin{array}{l}\text { Betriebe } \\
\text { Farms } \\
\text { (n) }\end{array}$} & \multirow{2}{*}{$\begin{array}{l}\text { Schläge } \\
\text { Fields } \\
\text { (n) }\end{array}$} & \multicolumn{3}{|c|}{$\begin{array}{c}\text { Meereshöhe / Altitude } \\
\text { (m) }\end{array}$} & \multicolumn{3}{|c|}{$\begin{array}{c}\text { Hangneigung / Slope } \\
\text { (\%) }\end{array}$} & \multicolumn{3}{|c|}{$\begin{array}{c}\text { Schlagfläche / Field size } \\
\text { (ha) }\end{array}$} & \multirow{2}{*}{$\begin{array}{l}\text { Erhobene Arbeitsgänge } \\
\text { Recorded field operations } \\
\text { (n) }\end{array}$} \\
\hline & & & $\min$ & $\mathrm{m}$ & $\max$ & $\min$ & $m$ & $\max$ & $\min$ & $m$ & $\max$ & \\
\hline 2011 & 19 & 100 & 808 & 1277 & 2084 & 3,5 & 34,9 & 85,8 & 0,1 & 1,8 & 8,2 & 3398 \\
\hline 2012 & 18 & 108 & 808 & 1318 & 2080 & 3,5 & 35,7 & 76 & 0,1 & 1,5 & 7,8 & 3886 \\
\hline 2013 & 19 & 109 & 808 & 1315 & 2079 & 3,5 & 35,7 & 76 & 0,1 & 1,6 & 7,8 & 3977 \\
\hline
\end{tabular}




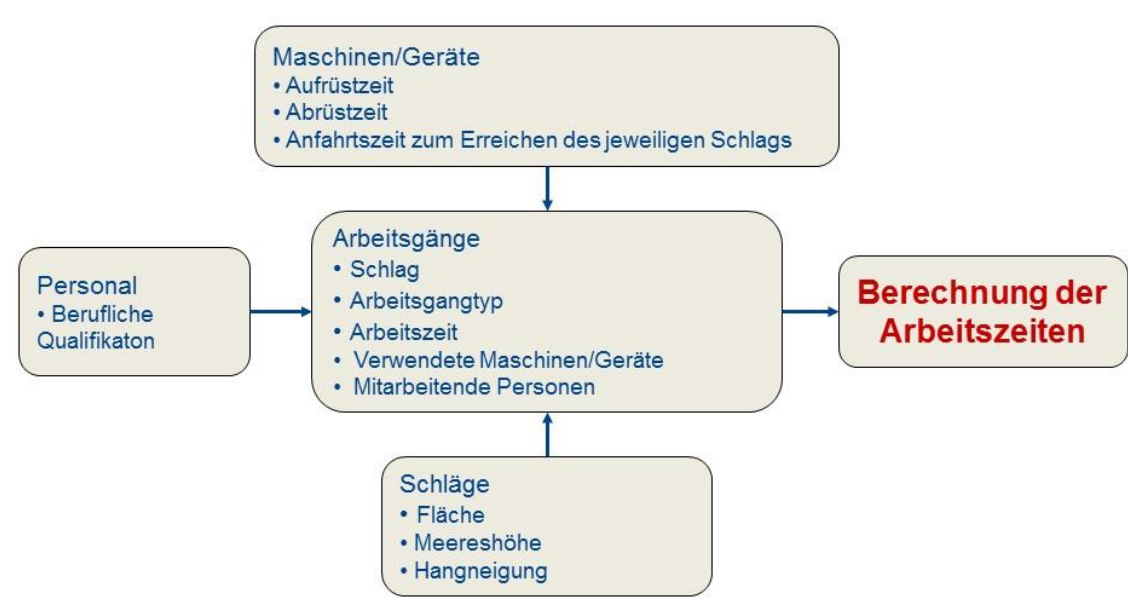

Abb. 2: Überblick der erhobenen Daten zur Schätzung der Arbeitszeiten bei der Futterproduktion // Overview of the data collected for the estimation of the labour requirements for forage production.

der Erhebung einzuholen und Rückfragen abzuklären. Die erfassten Daten wurden einer Plausibilitätsprüfung unterzogen, um eventuelle grobe Fehler während der Erhebung zu finden und auszubessern. Solche Fälle wurden zusammen mit der erhebenden Person besprochen. Daten, die mangelhaft, ungenau oder lückenhaft waren, wurden von der Auswertung ausgeschlossen. Zwei Betriebe standen nach dem ersten Jahr nicht mehr zur Verfügung, während bei einem anderen Betrieb die Daten des ersten Jahres aufgrund fehlerhafter Erfassung ausgeschlossen werden mussten. Ein weiterer Betrieb, der zu Beginn der Untersuchung in Form einer überbetrieblichen Kooperation organisiert war, wurde nach dem ersten Projektjahr in zwei eigenständige Betriebe aufgeteilt.

Insgesamt wurden während der drei Projektjahre 11261 einzelne Arbeitsgänge für die Kategorien Wiese und Mähweide erhoben (Tab. 2). Dazu kommen weitere $379 \mathrm{Ar}-$ beitsgänge für die Silomaisflächen, was eine Gesamtanzahl von 11640 Arbeitsgängen ergibt.

\section{CHARAKTERISIERUNG DES PRO- DUKTIONSSYSTEMS IM UNTER- SUCHTEN GEBIET}

Die untersuchten Grünlandflächen werden fast ausschließlich mit Wirtschaftsdüngern bei einem durchschnittlichen Viehbesatz von 1,8 Großvieheinheiten (GVE) pro Hektar gedüngt [7]. Nur bei 4,5\% der Arbeitsgänge ist ein zumindest teilweiser überbetrieblicher Personal- und Maschineneinsatz (vor allem Maschinenringeinsatz) vorhanden [7]. untergeordnete Rolle (Abb. 3). Sie wird vor allem bei den ersten zwei Schnitten (14\% der Gesamtproduktion beim ersten Schnitt,
$11 \%$ beim zweiten Schnitt) eingesetzt, ist sehr niedrig beim dritten Schnitt (2\%) und bleibt beim vierten Schnitt vollständig aus. Die Belüftung ist das am meisten eingesetzte Verfahren vom ersten bis zum dritten Schnitt (jeweils 68\%, 73\% und 66\% des geernteten Futters beim entsprechenden Schnitt), während sie beim vierten Schnitt nur $14 \%$ ausmacht. Die Silierung spiegelt diesen Trend in den ersten zwei Schnitten wider (jeweils $18 \%$ und $16 \%$ ). Sie stellt etwa ein Drittel (32\%) der Produktion beim dritten Schnitt dar und ist das am meisten eingesetzte Konservierungsverfahren beim vierten Schnitt (86\%). Die Eigenschaften der Betriebe, die am Projekt beteiligt waren, sind im Anhang (Tab. a) zusammengefasst.

\section{BERECHNUNG DES ARBEITSZEITBE- DARFS}

Beim Personal wurde für jede mitarbeitende Person die Arbeitszeit jedes einzelnen Ar beitsganges erhoben. Es wurden auch folgende Größen mitberücksichtigt:

- Aufrüstzeiten der Maschinen/Geräte, die für den Arbeitsgang eingesetzt wurden, wurden dem Betriebsleiter (wenn beim



Abb. 3: Anteil der Futterkonservierungsverfahren an der gesamten Futterproduktion der untersuchten Schläge bei den verschiedenen Schnitten ([8], abgeändert). Die angegebenen Anteile beziehen sich auf die Gesamtproduktion aller drei Beobachtungsjahre // Proportion of different forage conservation systems within each growth cycle on the total forage production ([8], adapted). The values refer to the total forage production across three observation years. 
Tab. 3: Aggregierung der ursprünglichen Beschreibungen der Arbeitsgänge in Makrokategorien und Anzahl der Beobachtungen // Aggregation of the original descriptions of field operations into macrocategories and number of observations.

\begin{tabular}{|c|c|c|c|c|c|c|c|}
\hline \multirow{2}{*}{$\begin{array}{l}\text { Nutzungsform } \\
\text { Utilisation form }\end{array}$} & \multirow{2}{*}{$\begin{array}{l}\text { Ursprüngliche Beschreibung des Arbeitsgangs } \\
\text { Original description of the operation }\end{array}$} & \multirow{2}{*}{$\begin{array}{l}\text { Makrokategorie } \\
\text { Macrocategory }\end{array}$} & \multicolumn{5}{|c|}{$\begin{array}{l}\text { Hangneigungsklasse } \\
\text { Slope class }\end{array}$} \\
\hline & & & $<20 \%$ & $20-40 \%$ & $40-60 \%$ & $>60 \%$ & $\begin{array}{l}\text { Gesamt } \\
\text { Total }\end{array}$ \\
\hline \multirow{7}{*}{ Wiese } & Düngen, Gülle rühren, Mist/Gülle aufladen & Düngung & 46 & 72 & 42 & 31 & 191 \\
\hline & $\begin{array}{l}\text { Feld säubern, Pflügen, Fräsen, Saatbettvor- } \\
\text { bereitung, Walzen, Neuansaat, Aussaat, Über- } \\
\text { saat/Nachsaat, Eggen, Striegeln, Unkrautbe- } \\
\text { kämpfung, Mäusebekämpfung }\end{array}$ & Grünlandpflege & 79 & 82 & 60 & 34 & 255 \\
\hline & $\begin{array}{l}\text { Mähen, Zetten, Wenden, Schwaden, Rechen, } \\
\text { Heutrocknung mit Gerüst }\end{array}$ & Handarbeit bei Futterernte & 78 & 87 & 77 & 48 & 290 \\
\hline & Mähen, Zetten, Wenden, Schwaden, Pressen/Wickeln & Mechanisierte Futterernte & 72 & 89 & 68 & 44 & 273 \\
\hline & Aufladen, Einfahren zur Hofstelle, Transport & Aufladen-Transport & 50 & 82 & 68 & 48 & 248 \\
\hline & Handarbeit im Stadel, Heukranarbeiten & Arbeit im Stadel & 60 & 72 & 71 & 17 & 220 \\
\hline & $\begin{array}{l}\text { Instandhaltung von Bächen/Waalen/Wegen/Zäunen/ } \\
\text { Mauern, Personentransport, Beregnen, Heubergung } \\
\text { mit Seilwinde/Tragenetzen/Heuschieber, Striegeln/ } \\
\text { Mähen, Häckseln, Silieren, Weidearbeit (Herbstweide), } \\
\text { Belüften }\end{array}$ & Ohne Kategoriezuordnung & 14 & 50 & 45 & 15 & 124 \\
\hline \multirow{7}{*}{ Mähweide } & Düngen, Gülle rühren, Mist/Gülle aufladen & Düngung & 0 & 0 & 0 & 0 & 0 \\
\hline & $\begin{array}{l}\text { Mähen, Zetten, Wenden, Schwaden, Rechen, } \\
\text { Heutrocknung mit Gerüst }\end{array}$ & Handarbeit bei Futterernte & 1 & 1 & 6 & 4 & 12 \\
\hline & Mähen, Zetten, Wenden, Schwaden, Pressen/Wickeln & Mechanisierte Futterernte & 0 & 3 & 4 & 4 & 11 \\
\hline & $\begin{array}{l}\text { Feld säubern, Pflügen, Fräsen, Saatbettvorbereitung, } \\
\text { Walzen, Neuansaat, Aussaat, Übersaat/Nachsaat, Eggen, } \\
\text { Striegeln, Unkrautbekämpfung, Mäusebekämpfung }\end{array}$ & Grünlandpflege & 1 & 2 & 5 & 2 & 10 \\
\hline & Handarbeit im Stadel, Heukranarbeiten & Arbeit im Stadel & 1 & 3 & 6 & 4 & 14 \\
\hline & $\begin{array}{l}\text { Instandhaltung von Bächen/Waalen/Wegen/Zäunen/ } \\
\text { Mauern, Personentransport, Beregnen, Heubergung } \\
\text { mit Seilwinde/Tragenetzen/Heuschieber, Striegeln/Mähen, } \\
\text { Häckseln, Silieren, Weidearbeit (Herbstweide), Belüften }\end{array}$ & Ohne Kategoriezuordnung & 0 & 1 & 2 & 1 & 4 \\
\hline & Weidearbeit & Weidearbeit & 1 & 2 & 6 & 4 & 13 \\
\hline \multirow{5}{*}{ Silomais } & $\begin{array}{l}\text { Pflügen, Feld säubern, Eggen/Striegeln, } \\
\text { Saatbettvorbereitung }\end{array}$ & Feldvorbereitung & 23 & 4 & 0 & 0 & 27 \\
\hline & Düngen & Düngung & 23 & 4 & 0 & 0 & 27 \\
\hline & Aussaat & Aussaat & 11 & 4 & 0 & 0 & 15 \\
\hline & Unkrautbekämpfung, Beregnung (mobil) & $\begin{array}{l}\text { Unkrautbekämpfung } \\
\text { und Beregnung }\end{array}$ & 22 & 4 & 0 & 0 & 26 \\
\hline & $\begin{array}{l}\text { Schneiden, Häckseln, Aufladen/Einfahren zur Hofstelle, } \\
\text { Transport, Pressen/Wickeln, Silieren }\end{array}$ & Ernte und Silierung & 5 & 0 & 0 & 0 & 5 \\
\hline
\end{tabular}

jeweiligen Arbeitsgang anwesend) oder einer spezialisierten Fachkraft zugeordnet.

- An- und Rückfahrtzeit: Für den Betriebsleiter (oder das fachbezogene Personal, das im Arbeitsschritt involviert war) wurde die doppelte Zeit (hin und zurück) mit der jeweils verwendeten, selbstfahrenden Maschine, für anderes Personal mit anderen Fahrzeugen hingegen das Doppelte der Standardanfahrtszeit verrechnet.
- Abrüstzeiten der Maschinen/Geräte, die für den Arbeitsgang eingesetzt wurden, wurden dem Betriebsleiter (wenn beim jeweiligen Arbeitsgang anwesend) oder einer spezialisierten Fachkraft zugeordnet. 


\section{DATENSATZVORBEREITUNG}

Die Auswertung erfolgte getrennt für die Kategorien Wiese, Mähweide und Silomais.

Für die Untersuchung des Effektes der Hangneigung auf die Arbeitszeiten bei der Kategorie Wiese wurden die Neigungsklassen nach [8] berücksichtigt: 0-20\%, 20-40\%, $40-60 \%,>60 \%$.

Aufgrund der sehr niedrigen Anzahl an Beobachtungen pro Hangneigungsklasse für die Mähweiden wurden zwei breitere Klassen $(0-40 \%$ und $>40 \%$ ) beim Faktor Hangneigung untersucht.

Bei der Silomaisnutzung wurde keine Differenzierung nach Hangneigungsklassen unternommen. In diesem Fall gehören die meisten Werte zur niedrigsten Hangneigungsklasse und die sehr geringe Anzahl an Werten in der zweiten Klasse stammen alle aus zwei Schlägen eines einzigen Betriebs.

Für die Auswertung ist der ursprüngliche Datenbestand [8] für den vorliegenden Zweck umstrukturiert und angepasst worden. Folgende Änderungen wurden unternommen:

- Arbeitsgänge mit fehlenden oder unvollständigen Angaben zur Arbeitszeit der involvierten Personen (typischerweise die Arbeitsgänge mit einer Kombination von überbetrieblichen Kosten und Fremdmaschineneinsatz/Maschinenringeinsatz) sind bei der Auswertung nicht berücksichtigt worden. Dadurch wurden insgesamt 525 Beobachtungen (4,5\% der Gesamtanzahl) aus dem auszuwertenden Datenbestand ausgeschlossen, was zu einer Anzahl von 11115 gültigen Einzelbeobachtungen führte. Es ist hier anzumerken, dass die fehlenden Werte, die sich aufgrund der überbetrieblichen Kosten und des Fremdund Maschinenringeinsatzes ergeben, einen verhältnismäßig großen Verlust an Beobachtungen vor allem bei der niedrigsten Neigungsklasse bewirken, da bei dieser der überbetriebliche Maschineneinsatz häufiger ist. Insgesamt konnten 217 Beobachtungen in der Klasse $0-20 \%$ $(35,2 \%$ der Beobachtungen in dieser Klasse), 94 in der Klasse 20-40\% (14,9\% der Beobachtungen in dieser Klasse), 115 in der Klasse 40-60\% (21,1\% der Beobachtungen in dieser Klasse) und 99 in der Klasse $>60 \%$ (29,4\% der Beobachtungen in dieser Klasse) somit nicht berücksichtigt werden.

- Um eine aussagekräftige Anzahl an Wiederholungen pro Neigungsklasse und
Arbeitskategorie zu erhalten, sind die ursprünglich angegebenen Beschreibungen der Arbeitsgänge zu einer überschaubaren Anzahl von Arbeitsmakrokategorien (ab hier als Makrokategorien bezeichnet) zugeordnet worden (Tab. 3). Einige Arbeitsgänge, die ursprünglich in dieselbe Arbeitskategorie (Rechen, Mähen, Zetten, Wenden, Schwaden) hineinfielen, wurden zur Makrokategorie „Handarbeit bei Futterernte“ oder "Mechanisierte Futterernte" zugeordnet (je nachdem, ob im Einzelfall Maschinen im Einsatz waren).

Bei der Auswertung der durchschnittlichen Arbeitszeiten jeder einzelnen Makrokategorie sind all jene Schläge herangezogen worden, bei denen die jeweilige Makrokategorie vertreten war

- Für die Auswertung der durchschnittlichen Gesamtarbeitszeiten wurde ein Schlag von der Auswertung ausgeschlossen, sobald ein einziger Arbeitsgang unvollständige Arbeitszeiten aufwies.

- Werte, welche ursprünglich zur Kategorie Weidearbeit zugeordnet wurden, wurden der gleichnamigen Makrokategorie bei den Mähweiden zugeordnet, wenn es sich um Schläge mit höchstens einer Mahd pro Jahr und vorwiegender Weidenutzung in der restlichen Saison handelte. Somit wurde die Herbstweide bei dieser Makrokategorie nicht berücksichtigt.

Alle Makrokategorien der Nutzungsform Wiese sind im Datenbestand mit 191 bis 290 Fällen gut vertreten (Tab. 3). Bei den Mähweideflächen ist die Anzahl der Beobachtungen sehr niedrig (4-14) und die Kategorie Düngung fehlt vollständig, weil überbetriebliche Kosten bei allen Arbeitsgängen vorhanden waren. Bei der Nutzungsform Silomais ist eine etwas höhere Anzahl an Fällen (2627) für Feldvorbereitung, Düngung sowie Unkrautbekämpfung und Beregnung zu verzeichnen. Für Aussaat (15) und vor allem für Ernte und Silierung (5) ist die Anzahl der Beobachtungen wesentlich niedriger, da es sich um Arbeitsgänge handelt, bei denen der
Maschinenringeinsatz oder eine andere Form des überbetrieblichen Maschineneinsatzes sehr oft in Anspruch genommen wurde.

Für die Auswertung des Gesamtarbeitszeitbedarfs sind insgesamt 197 gültige Fälle für die Wiesen verfügbar (Tab. 4). Dabei ist die Neigungsklasse $<20 \%$ etwas unterrepräsentiert. Der Grund dafür ist vor allem der häufigere überbetriebliche Maschineneinsatz in dieser Neigungsklasse, was zum Ausschluss der entsprechenden Beobachtungen führte.

Da aufgrund des überbetrieblichen Einsatzes in zumindest einer der Makrokategorien nur zwei auswertbare Fälle für die Mähweiden und eine für die Silomaisflächen verfügbar sind, ist die Auswertung der Daten dieser Nutzungsformen nicht sinnvoll.

\section{STATISTISCHE AUSWERTUNG}

Innerhalb jeder Makrokategorie wurde der Effekt der Hangneigungsklasse auf den Arbeitszeitbedarf mittels gemischter Modelle (Prozedur MIXED der Software IBM SPSS Statistics 25 (IBM, Armonk, New York, USA)) untersucht. Dabei wurden der Betrieb und seine Wechselwirkung mit dem Jahr als zufällige Effekte betrachtet. Die wiederholten Messungen über die Jahre bei demselben Schlag wurden ebenfalls im Modell berücksichtigt. Die Kovarianzstruktur der wiederholten Messungen wurde mittels AIC (Akaike's Information Criterion) angepasst. Mittelwertvergleiche wurden mittels Least Significant Difference durchgeführt.

Der Gesamtarbeitszeitbedarf wurde als Funktion der Meereshöhe und der Hangneigung mittels gemischter Modelle unter Berücksichtigung derselben Designeffekte (Betrieb und seine Wechselwirkung mit dem Jahr als zufällige Faktoren, Berücksichtigung der wiederholten Messungen über die Jahre bei demselben Schlag) untersucht. Der Effekt der Schlagfläche wurde im Modell nicht berücksichtigt, weil sie ursprünglich keine

Tab. 4: Anzahl der gültigen Beobachtungen für die Auswertung des Gesamtarbeitszeitbedarfs je nach Nutzungsform // Number of available observations for the analysis of the total labour requirements according to the agricultural use.

\begin{tabular}{lccc|cc} 
Nutzungsform & \multicolumn{5}{c}{ Hangneigungsklasse / Slope class } \\
Utilisation form & $<20 \%$ & $\mathbf{2 0 - 4 0 \%}$ & $\mathbf{4 0 - 6 0 \%}$ & $>\mathbf{6 0 \%}$ & Gesamt / Total \\
Wiese & 30 & 65 & 55 & 47 & 197 \\
Mähweide & 0 & 0 & 1 & 1 & 2 \\
Silomais & 1 & 0 & 0 & 0 & 1
\end{tabular}


Zielgröße bei der Erstellung des Untersuchungsplans darstellte, eine asymmetrische Verteilung aufweist (kleine Schläge sind am stärksten im Datenbestand vertreten) und hochsignifikant (obwohl nicht eng) mit der Hangneigung ( $R=-0,436$ nach Spearman, $P<0,01)$ korreliert ist. Das Modell wurde ausgehend vom Basismodell mit den Designeffekten schrittweise vorwärts unter Verwendung vom Maximum Likelihood (ML) unter Verwendung von AIC als Indikator für die Anpassungsgüte sukzessiv aufgebaut. Das endgültige Modell wurde dann mittels Restricted Maximum Likelihood (REML) berechnet und seine Anpassungsgüte wurde mittels fünffacher Kreuzvalidierung [16] in Form des quadrierten Korrelationskoeffizienten nach Pearson zwischen beobachteten und aufgrund des fixen Teils des Modells vorausgesagten Werten in SAS 9.2 (SAS
Institute Inc., Cary, North Carolina, USA) für Linux geschätzt.

Bei allen Analysen wurde die Normalverteilung der Residuen und die Varianzhomogenität grafisch überprüft [17]. Wenn die Daten die Annahmen nicht erfüllten, wurden geeignete Datentransformationen durchgeführt. Mittelwerte von Auswertungen mit transformierten Daten werden als rücktransformierte Werte angezeigt, mit Ausnahme derjenigen im Anhang (Tab. b, Tab. c, Tab. d).

Bei fehlender Erfüllung der Annahmen auch nach der Datentransformation, wie im Fall der stark nichtbalancierten Daten der Mähweiden, wurde der nichtparametrische Test nach Kruskal-Wallis verwendet.

Eine Signifikanzgrenze von $\alpha=0,05$ wurde für alle Analysen verwendet.
Die Ergebnisse, die grafisch dargestellt werden, werden zusätzlich in numerischer Form unter Angabe relevanter beschreibender Größen der Datenverteilung im Anhang (Tab. b, Tab. c, Tab. d) angegeben.

Um neben Hangneigung und Meereshöhe die Relevanz einiger zusätzlicher Einflussgrößen explorativ zu erkunden, die zur Erstellung des Untersuchungsplans ursprünglich nicht berücksichtigt wurden, aber vermutlich eine Rolle in der landwirtschaftlichen Praxis spielen (Anzahl mitarbeitender Personen pro Arbeitsgang und ha, nur Handarbeit bei Futterernte, Entfernung des Schlags von den Hofgebäuden, Schlagfläche in ha, Nutzungshäufigkeit in Nutzungen pro Jahr inkl. der Herbstweide und Schnitthäufigkeit), wurde zusätzlich eine Ordination der Daten mittels Hauptkomponentenanalyse (PCA) durchgeführt. Für jede Variable wurde von

Tab. 5: Effekt der Hangneigungsklassen auf den Arbeitszeitbedarf in AKh/ha verschiedener Makrokategorien und auf den Gesamtarbeitszeitbedarf bei Wiesennutzung. Analyse mittels gemischter Modelle. $P=$ Wahrscheinlichkeit des Effektes der Hangneigungsklasse (signifikante Effekte sind fett markiert), $\mathrm{N}=$ Anzahl der Beobachtungen, $\mathrm{MW}$ = rücktransformierter Randmittelwert. Werte ohne gemeinsame Buchstaben unterscheiden sich signifikant voneinander. Auswertung mit transformierten Daten: \# Logarithmus zur Basis $10,{ }^{+}$Quadratwurzel, ${ }^{*}$ natürlicher Logarithmus // Effect of slope classes on the labour requirements in labour hours per hectare for different macrocategories and on the total labour re quirements for meadows. Data analysis by means of mixed models. $P=$ probability of the effect of the slope classes (significant effects are highlighted in bold), $N=$ number of observations, MW = back-transformed estimated marginal means. Values sharing no superscript letters significantly differ from each other. Analysis with transformed data: \# base 10 logarithm, † square root, ¥ natural logarithm.

\begin{tabular}{|c|c|c|c|c|c|c|}
\hline \multirow{2}{*}{$\begin{array}{l}\text { Makrokategorie } \\
\text { Macrocategory }\end{array}$} & \multirow{2}{*}{$\mathbf{P}$} & \multirow{2}{*}{$\begin{array}{l}\text { Parameter } \\
\text { Parameter }\end{array}$} & \multicolumn{4}{|c|}{ Hangneigungsklasse / Slope class } \\
\hline & & & $<20 \%$ & $20-40 \%$ & $40-60 \%$ & $>60 \%$ \\
\hline \multirow{2}{*}{ Düngung\# } & \multirow{2}{*}{0,458} & $\mathrm{~N}$ & 46 & 72 & 42 & 31 \\
\hline & & MW & 4,7 & 4,8 & 5,2 & 7,1 \\
\hline \multirow{2}{*}{ Grünlandpflege ${ }^{\#}$} & \multirow{2}{*}{$<0,001$} & $\mathrm{~N}$ & 79 & 82 & 60 & 34 \\
\hline & & MW & $2,0^{a}$ & $2,9 \mathrm{ab}$ & $4,1^{\mathrm{b}}$ & $7,6^{c}$ \\
\hline \multirow{2}{*}{ Handarbeit bei Futterernte ${ }^{\#}$} & \multirow{2}{*}{$<0,001$} & $\mathrm{~N}$ & 78 & 87 & 77 & 47 \\
\hline & & MW & $8,0^{\text {a }}$ & $12,3^{b}$ & $34,6^{c}$ & $46,0^{c}$ \\
\hline \multirow{2}{*}{ Mechanisierte Futterernte ${ }^{\dagger}$} & \multirow{2}{*}{0,973} & $\mathrm{~N}$ & 72 & 89 & 68 & 44 \\
\hline & & MW & 13,8 & 14,4 & 14,5 & 14,4 \\
\hline \multirow{2}{*}{ Aufladen-Transport $\#$} & \multirow{2}{*}{0,003} & $\mathrm{~N}$ & 50 & 81 & 68 & 48 \\
\hline & & MW & $3,4^{\text {a }}$ & 4,4 ab & $5,0^{b c}$ & $6,3^{c}$ \\
\hline \multirow{2}{*}{ Arbeit im Stadel\# } & \multirow{2}{*}{$<0,001$} & $\mathrm{~N}$ & 60 & 72 & 71 & 17 \\
\hline & & MW & $1,8^{\text {a }}$ & $2,3^{b}$ & $2,5^{b}$ & $1,3^{a}$ \\
\hline \multirow{2}{*}{ Ohne Kategoriezuordnung\# } & \multirow{2}{*}{0,044} & $\mathrm{~N}$ & 14 & 50 & 45 & 15 \\
\hline & & MW & $2,4^{a}$ & $4,3 \mathrm{ab}$ & $4,8^{b}$ & $8,0^{b}$ \\
\hline \multirow{2}{*}{ Gesamtarbeitszeitbedarf ${ }^{\sharp}$} & \multirow{2}{*}{$<0,001$} & $\mathrm{~N}$ & 29 & 63 & 54 & 46 \\
\hline & & MW & $38,8^{a}$ & $51,5^{\text {a }}$ & $71,4^{b}$ & 92,9 b \\
\hline
\end{tabular}


jedem Wert der Mittelwert abgezogen und das Ergebnis durch die Standardabweichung dividiert (z-Transformation), so dass die resultierende Variable einen Mittelwert von Null und eine Varianz von Eins besitzt.

\section{SCHÄTZUNG DES ARBEITSZEITBE-}

\section{DARFS DER UNTERSUCHTEN BE-}

\section{TRIEBE NACH SCHICK (1995) [10]}

Für die Schweiz kann der Arbeitszeitbedarf von Viehwirtschaftsbetrieben nach der Gleichung von Schick [10] geschätzt werden. Der Arbeitszeitbedarf wird auf Betriebsebene pro gehaltener Rinder-GVE anhand des Flächenanteils mit mehr als 30\% Hangneigung, der durchschnittlichen Schlagfläche, der durchschnittlichen Meereshöhe, der Schnitthäufigkeit sowie der Anzahl an gehaltenen GVE über eine Regressionsgleichung geschätzt. Für die Schätzung des Arbeitszeitbedarfs nach Schick [10] wurden alle teilnehmenden Betriebe herangezogen, deren Futterflächen nahezu vollständig erhoben und mindestens zwei Jahre lang untersucht wurden. Eine maximale Abweichung von 0,5 ha oder $10 \%$ im Vergleich zur effektiven Gesamtfutterfläche wurde toleriert. Zudem wurden jene Betriebe aus der Berechnung ausgeschlossen, bei welchen die Ausprägung der Einflussgrößen außerhalb des Kalibrationsbereichs (gehaltene GVE: 5-38; durchschnittliche Meereshöhe der Futterfläche: 630-1700 m; prozentueller Flächenanteil mit Hangneigung >35\%: $0-84 \%$; durchschnittliche Schlagfläche: 0,5-5,6 ha) lag. Abweichungen von $\pm 5 \%$ wurden bei allen Kalibrationsintervallen toleriert. Alle notwendigen Einflussgrößen wurden aus dem vorhandenen Datensatz entnommen und in die Gleichung eingesetzt. Da das Ergebnis Arbeitskraftstunden pro GVE (AKh/GVE) ergab, war eine Transformation in $\mathrm{AKh} / \mathrm{ha}$ notwendig. Zu diesem Zweck wurde das Ergebnis mit der Anzahl an gehaltenen GVE multipliziert und durch die gesamte Futterfläche dividiert. Im Fall eines externen Futterzukaufs wurde die Futterfläche um einen Wert erhöht, der sich aus dem Verhältnis des Gewichts der Trockenmasse (TM) des zugekauften Futters und TM-Futterertrag/ha des Betriebs aufgrund der Meereshöhe nach [8] ergab. Für diese Gleichung zur Berechnung des TM-Futterertrags wurden die Daten der vorliegenden Untersuchung genutzt und der TM-Futterertrag in Abhängigkeit der Meereshöhe geschätzt. Als Meereshöhe des Betriebs wurde die mittlere Meereshöhe der untersuchten Schläge, gewichtet nach der Fläche, herangezogen.

\section{ERGEBNISSE}

\section{WIESEN}

\section{ARBEITSZEITBEDARF IN VERSCHIEDE- NEN MAKROKATEGORIEN}

Bei den Wiesen war vor allem die starke Zunahme der Handarbeit bei der Futterernte mit zunehmender Hangneigung auffallend (Tab. 5). Dabei betrug der Arbeitszeitbedarf bei der höchsten Neigungsklasse mehr als das Fünffache der niedrigsten Neigungsklasse. Ein zunehmender Trend war auch für die mechanisierte Futterernte, die Grünlandpflege sowie Aufladen-Transport grafisch erkennbar.

Die statistische Analyse zeigte hingegen keinen signifikanten Effekt der Hangneigungsklasse auf den Arbeitszeitbedarf für Düngung und mechanisierte Futterernte (Tab. 5). Der Arbeitszeitbedarf aller anderen Makrokategorien wurde davon signifikant beeinflusst und nahm mit zunehmender Neigungsklasse zu. Eine Ausnahme bildete die Arbeit im Stadel. Es ist denkbar, dass die Arbeitszeiten dieser Kategorie in Folge geringerer Erträge bei der höchsten Neigungsklasse etwas niedriger sind, weil die ungünstige Topografie die Düngung erschwert und zu verringerten Erträgen mit entsprechend reduziertem Aufwand bei der Futteraufbereitung und Futterbewegung führt.

Die starke Zunahme des Arbeitszeitbedarfs vor allem in der Makrokategorie Handarbeit bei Futterernte scheint auch von der Tatsache beeinflusst zu sein, dass bei den topografisch ungünstigen Flächen eine zunehmende Anzahl an mitarbeitenden Personen tätig ist, um die gewünschte Schlagkraft zu erreichen. Die durchschnittliche Anzahl an mitarbeitenden Personen pro Hektar zeigt



Makrokategorie

-Düngen

- Grünlandpflege

Handarbeit bei

Futterernte

- Mechanisierte Futterernt

Aufladen - Transport

- Arbeit im Stadel

Ohne

Kategoriezuordnung

Fehlerbalken: +l-1 SE

Abb. 4: Durchschnittliche Anzahl an mitarbeitenden Personen pro Arbeitsgang und Hektar bei verschiedenen Hangneigungsklassen // Average workers number per operation and hectare for different slope classes. 
gerade in dieser Kategorie eine mehr als vierfache Zunahme von der günstigsten hin zur ungünstigsten Hangneigungsklasse (Abb. 4). Eine Zunahme ist auch für die meisten anderen Kategorien (zumindest von der ersten bis zur dritten Hangneigungskategorie) grafisch erkennbar, aber sie ist bei weitem nicht so ausgeprägt wie im Fall der Handarbeit bei Futterernte. Die Korrelation nach Pearson des Gesamtarbeitszeitbedarfs mit der durchschnittlichen Anzahl an mitarbeitenden Personen war für alle Kategorien hochsignifikant $(P<0,001)$, aber für die meisten Kategorien sehr schwach bis relativ schwach ( $R=0,322$ für die Arbeit im Stadel, $R=0,404$ für Aufladen-Transport, $R=0,413$ für Ohne Kategoriezuordnung, $R=0,459$ für Düngen). Die höchste Korrelation wurde für die Handarbeit bei Futterernte $(R=0,616)$ gefolgt von der Grünlandpflege $(R=0,614)$ beobachtet.

\section{GESAMTARBEITSZEITBEDARF}

Die vorwärts stufenweise lineare Regressionsanalyse mittels gemischter Modelle zeigte beim ersten Schritt der Modellentwicklung einen hochsignifikanten Effekt $(P<0,001)$ der Hangneigung auf den Gesamtarbeitszeitbedarf (Analyse mit logarithmier- ten Werten). Dieses Modell konnte in weiteren Schritten nicht weiter verbessert werden. Beim Test eines Polynoms zweiten Grades war der quadratische Term nicht signifikant $(P=0,093)$. Die Meereshöhe war ebenfalls beim ersten Schritt nicht signifikant $(P=0,155)$. Dieses Ergebnis deckt sich gut mit den Erkenntnissen aus der Betrachtung der Produktionskosten (aus dem vorliegenden Datenbestand berechnet), welche zum Teil von den Arbeitszeiten abhängen: kein Effekt der Meereshöhe war auf die Kosten pro Hektar zu verzeichnen, während bei der Berechnung der Kosten in Bezug auf den Trockenmasse-Ertrag ihr Effekt offensichtlich wurde [7]. In der Literatur wird ein Effekt der Meereshöhe auf die Kosten pro Hektar erkannt, der aber in verschiedene Richtungen läuft: Laut Schick [10] nimmt der Gesamtarbeitszeitbedarf pro gehaltener GVE mit der Höhe zu, während andere Quellen ([4] [13]) schätzen, dass die Arbeitszeiten pro ha bei einer Reduktion der Schnitthäufigkeit abnehmen. Da in der Tendenz die Schnitthäufigkeit mit zunehmender Meereshöhe abnimmt, ist es naheliegend, dass laut dieser Autoren die Kosten mit zunehmender Meereshöhe abnehmen sollten.
Aus der vorliegenden Untersuchung ergibt sich, dass nur die Hangneigung den Gesamtarbeitszeitbedarf beeinflusst (Abb. 5). Dabe betragen bei fehlender Neigung die Arbeitszeiten etwa $30 \mathrm{AKh} / \mathrm{ha}$, welche auf $75 \mathrm{AKh} /$ ha bei $50 \%$ Hangneigung und auf $125 \mathrm{AKh} /$ ha bei $80 \%$ Hangneigung ansteigen.

EXPLORATIVE ANALYSE DES ZUSAMMENHANGS ZWISCHEN DEM GESAMTARBEITSZEITBEDARFS MIT WEITEREN EINFLUSSGRÖßEN

Die ersten zwei Achsen der PCA, welche im Gegensatz zur Regressionsanalyse zusätzlich untersuchte Einflussgrößen (Anzahl mitarbeitender Personen pro Arbeitsgang und ha - nur Handarbeit bei Futterernte, Entfernung des Schlags von den Hofgebäuden, Schlagfläche, Nutzungshäufigkeit in Nutzungen pro Jahr inkl. der Herbstweide und Schnitthäufigkeit) berücksichtigt, erklären insgesamt $68,3 \%$ der Variabilität der Daten (Abb. 6). Achse 1 ist eng mit der Standardentfernung von Hof und der Meereshöhe positiv und korreliert mit der Nutzungs- und Schnitthäufigkeit, aber diese Achse zeigt kaum einen Zusammenhang mit dem $\mathrm{Ar}$ beitszeitbedarf. Achse 2 deutet hingegen

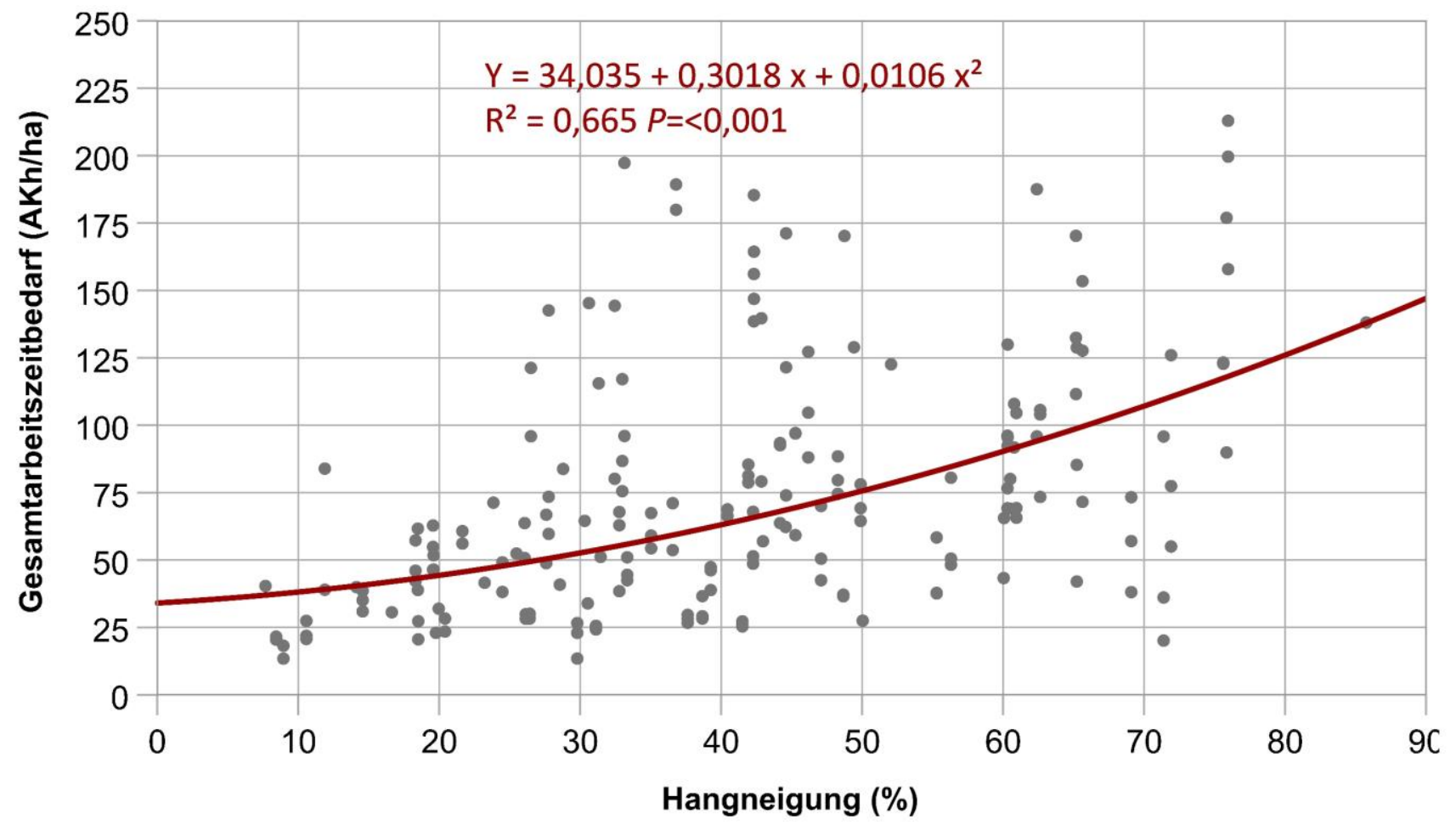

Abb. 5: Effekt der Hangneigung auf den Gesamtarbeitszeitbedarf bei Wiesen. Analyse mit logarithmierten Werten. Die rücktransformierte Funktion sowie die Originalwerte werden angezeigt. // Effect of slope inclination on the total labour requirements for meadows. Analysis with logarithmic values. The back-transformed function is displayed against the original values. 


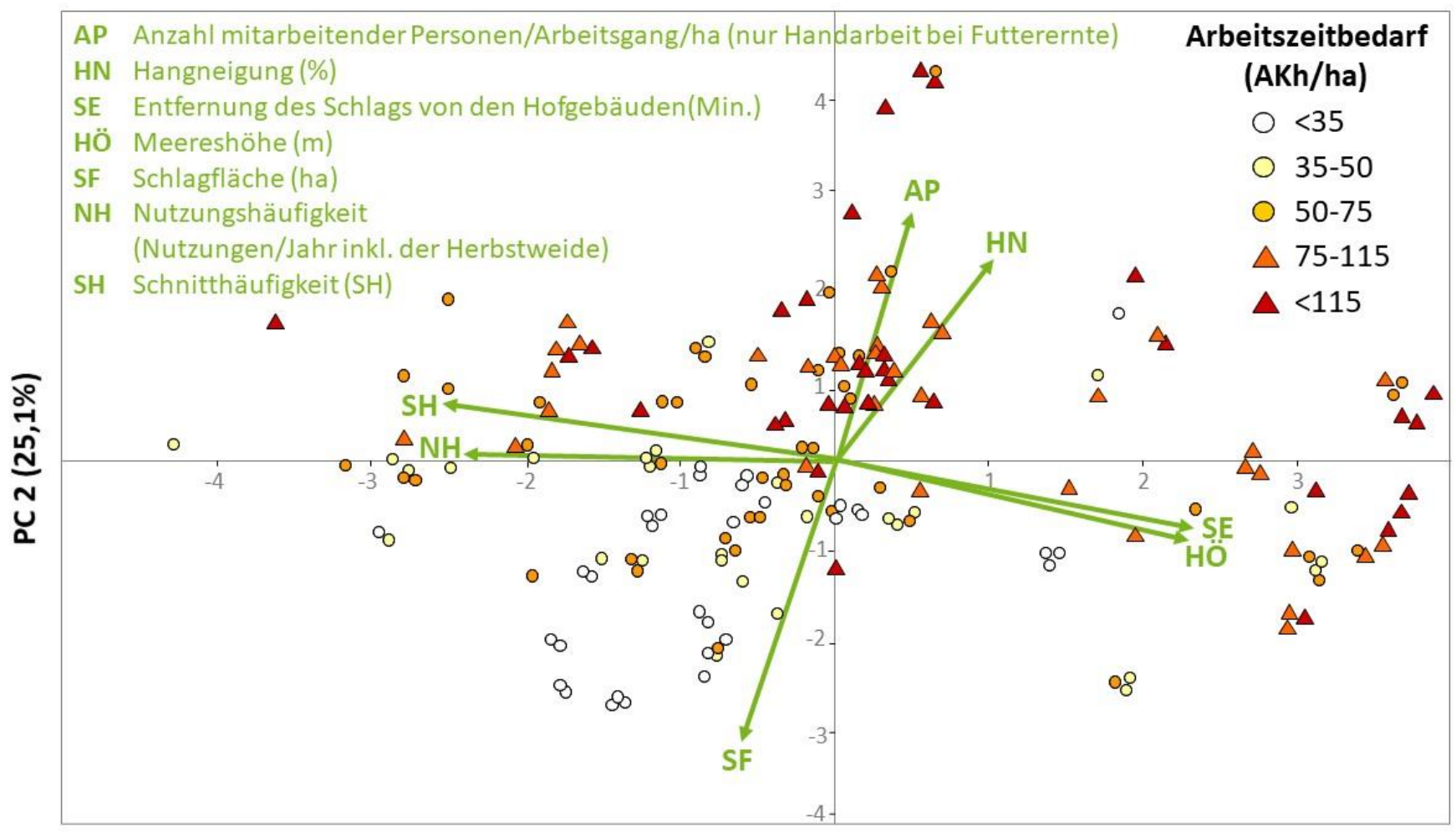

PC $1(43,2 \%)$

Abb. 6: Ordinationsdiagramm (biplot) aus Hauptkomponentenanalyse (PCA) der untersuchten Schläge in der Kategorie Wiese (als Klassen des Arbeitszeitbedarfs angezeigt) und einer Auswahl an Einflussgrößen, welche bei der Erstellung des ursprünglichen Untersuchungsplans nicht berücksichtigt wurden. // Ordination diagram (biplot) from Principal Component Analysis (PCA) of the investigated fields in the meadow category (displayed according to classes of labour requirements) and a selection of influencing variables that were not considered when the original investigation plan was drawn up.

auf einen positiven Zusammenhang des Arbeitszeitbedarfs mit der Hangneigung und mit der Anzahl mitarbeitender Personen pro Arbeitsgang und Hektar und auf einen negativen Zusammenhang mit der Schlagfläche hin, was mit den Ergebnissen anderer Studien des Arbeitszeitbedarfs für den Futterbau in der Schweiz [5] [10] übereinstimmt. Es darf aber auch nicht vergessen werden, dass Aufrundungen der Arbeitszeiten seitens der Beobachter bei sehr kleinen Schlägen leicht zu einer starken Überschätzung derselben führen.

\section{MÄHWEIDEN}

Der Effekt der Hangneigungsklasse war auch bei der grafischen Zusammenfassung des Arbeitszeitbedarfs bei der Mähweidenutzung offensichtlich (Tab. 6). Es ist auffallend, dass, wie bei den Wiesen, vor allem die Handarbeit bei der Futterernte die größte quantitative Änderung bei zunehmender Hangneigungsklasse von im Durchschnitt 1,2 zu etwa $60 \mathrm{AKh} / \mathrm{ha}$ aufweist, obwohl es sich um einen einzigen Schnitt handelt.
Es ist nochmals anzumerken, dass Werte für die Kategorie Düngung aufgrund des Vorhandenseins von überbetrieblichen Kosten bei allen beobachteten Arbeitsgängen vollständig fehlen und daher nicht dargestellt werden können. Das verhindert auch die Berechnung des gesamten Arbeitszeitbedarfs.

Der Arbeitszeitbedarf aller Makrokategorien mit Ausnahme der mechanisierten Futterernte und der Kategorie Ohne Kategoriezuordnung unterschiedet sich signifikant in Abhängigkeit der Hangneigungsklasse und war deutlich höher bei Hangneigungen von über $40 \%$.

Während die relativ großen Unterschiede beim Arbeitszeitbedarf für Weidearbeit, mechanisierte Futterernte, Handarbeit für die Futterernte sowie Aufladen und Transport nachvollziehbar sind, scheint vor allem die wesentlich größeren Arbeitszeiten im Stadel bei den höheren Hangneigungsklassen kaum erklärbar. Mit nur zwei Betrieben, welche sich in ihrer Flächenausstattung hinsichtlich der Hangneigung stark unterscheiden, besteht tatsächlich die Gefahr, dass die
Arbeitszeiten eher eine unterschiedliche Ausprägung der Arbeitseffizienz widerspiegeln als einen Effekt der Hangneigung.

Aus diesem Grund sind die Richtwerte für die Mähweidenutzung eher mit Vorsicht anzuwenden.

\section{SILOMAIS}

Mit etwa $12 \mathrm{AKh} / \mathrm{ha}$ war Ernte und Silierung die zeitaufwändigste Kategorie beim Silomaisanbau (Abb. 7), gefolgt von der Feldvorbereitung (Boden- und Saatbettvorbereitung) mit knapp $8 \mathrm{AKh} / \mathrm{ha}$. Die Düngung lag bei etwa $3 \mathrm{AKh} / \mathrm{ha}$, während Aussaat und Unkrautbekämpfung etwa $2 \mathrm{AKh} / \mathrm{ha}$ in Anspruch nahmen.

Obwohl die Berechnung des Gesamtarbeitszeitbedarfs über die einzelnen Schläge aufgrund des häufigen überbetrieblichen Einsatzes nicht möglich war (nur ein verfügbarer Schlag ohne überbetrieblichen Einsatz vorhanden), lässt sich aufgrund der Durchschnittswerte der verschiedenen Kategorien ein Gesamtarbeitszeitbedarf von etwa $27 \mathrm{AKh} /$ ha grob schätzen. Das entspricht 
Tab. 6: Effekt der Hangneigungsklassen auf den Arbeitszeitbedarf verschiedener Arbeitskategorien bei Mähweidenutzung. Analyse mittels gemischter Modelle außer \# = Analyse mittels KruskalWallis-Test. $\mathrm{P}=$ Wahrscheinlichkeit des Effektes der Hangneigungsklasse (signifikante Effekte sind fett markiert), $\mathrm{N}=$ Anzahl der Beobachtungen, $\mathrm{MW}=$ Mittelwert // Effect of slope classes on la bour requirements for different labour categories for grassland. Analysis by mixed models except $\#=$ analysis by Kruskal-Wallis-test. $P=$ probability of the effect of the slope class (significant effects are highlighted in bold), $N=$ number of observations, $M W=$ mean

\begin{tabular}{|c|c|c|c|c|}
\hline \multirow{2}{*}{$\begin{array}{l}\text { Arbeitskategorie } \\
\text { Work category }\end{array}$} & \multirow[t]{2}{*}{$\mathbf{P}$} & \multirow{2}{*}{$\begin{array}{l}\text { Parameter } \\
\text { Parameter }\end{array}$} & \multicolumn{2}{|c|}{$\begin{array}{l}\text { Hangneigungsklasse } \\
\text { Slope class }\end{array}$} \\
\hline & & & $0-40 \%$ & $>40 \%$ \\
\hline \multirow{2}{*}{ Grünlandpflege } & \multirow{2}{*}{0,017} & $\mathrm{~N}$ & 3 & 7 \\
\hline & & MW & 0,7 & 5,4 \\
\hline \multirow{2}{*}{ Weidearbeit } & \multirow{2}{*}{0,007} & $\mathrm{~N}$ & 3 & 10 \\
\hline & & MW & 2,1 & 10,9 \\
\hline \multirow{2}{*}{ Mechanisierte Futterernte } & \multirow{2}{*}{0,085} & $\mathrm{~N}$ & 3 & 8 \\
\hline & & MW & 4,6 & 21,9 \\
\hline \multirow{2}{*}{ Handarbeit bei Futterernte } & \multirow{2}{*}{0,030} & $\mathrm{~N}$ & 2 & 10 \\
\hline & & MW & 1,2 & 60,2 \\
\hline \multirow{2}{*}{ Aufladen-Transport } & \multirow{2}{*}{0,049} & $\mathrm{~N}$ & 3 & 10 \\
\hline & & MW & 1,4 & 7,7 \\
\hline \multirow{2}{*}{ Arbeit im Stadel } & \multirow{2}{*}{0,024} & $\mathrm{~N}$ & 3 & 10 \\
\hline & & MW & 0,8 & 6,2 \\
\hline \multirow{2}{*}{ Ohne Kategoriezuordnung ${ }^{\#}$} & \multirow{2}{*}{0,500} & $\mathrm{~N}$ & 1 & 3 \\
\hline & & MW & 0,5 & 6,5 \\
\hline
\end{tabular}

etwa der Arbeitszeit bei den Wiesenflächen mit einer Hangneigung von unter $20 \%$.

\section{DISKUSSION UND SCHLUSSFOL- GERUNGEN}

Es fällt auf, dass die ermittelten Richtwerte wesentlich höher sind als jene, welche in der verfügbaren Literatur im Alpenraum ([4] [13] [14]) geschätzt wurden (Abb. 8). Mögliche Gründe dafür sind:

- Im Vergleich zur oben genannten Literatur, bei der der Arbeitszeitbedarf aus der Summierung der Standardzeiten einer Reihenfolge von Operationen berechnet wird, schließen die Daten der vorliegenden Studie die Realität der untersuchten Betriebe (inkl. ihrer eventuellen Besonderheiten, betrieblicher Einschränkungen oder Ineffizienzen, aber auch der Verschnaufpausen bei körperlich anstrengenden Arbeiten, welche nur bei Schick [10] berücksichtigt werden) mit ein. Das ist vermutlich der Hauptgrund für die hohen Werte. Diese Vermutung wird durch die
Schätzung des Arbeitszeitbedarfs der untersuchten Betriebe nach der Gleichung von Schick [10] bestärkt, welche Ergebnisse in derselben Größenordnung der vorliegenden Studie liefert (Abb. 8).
Aufgrund der Konstellation der berücksichtigten Einflussgrößen sowie der Methodik für die Erhebung der Arbeitszeiten (Auswertung der Arbeitstagebücher der Landwirte) hat die Arbeit von Schick [10] eine gewisse Ähnlichkeit mit der vorliegenden Studie.

- Die sehr hohen Werte, welche bei den stark geneigten Wiesen- und Mähweideflächen beobachtet wurden und vor allem auf die Arbeitskategorie Handarbeit bei Futterernte zurückzuführen sind, ergeben sich unter anderem durch die Tatsache, dass bei solchen Flächen der Einsatz einer großen Erntemannschaft üblich ist, wie die Zunahme der durchschnittlichen Anzahl an mitarbeitenden Personen pro Hektar mit zunehmender Hangneigung zeigt (Abb. 4). Es ist hier anzumerken, dass alle mitwirkenden Personen (einschließlich der jüngeren und älteren Familienmitglieder, aber auch der freiwilligen Erntehelfer) in der Arbeitszeiterfassung erhoben wurden. Während in der Studie der Kosten der Futterproduktion, bei der dieselbe Datenbasis herangezogen wurde, symbolische Stundenkosten diesen Arbeiterkategorien zugewiesen wurden [8], ist es bei der vorliegenden Studie nicht möglich gewesen, ihren Beitrag zu gewichten, obwohl eine verminderte Arbeitseffizienz in manchen Fällen zu erwarten ist. Alles in allem ist es allerdings naheliegend, dass die Hauptstrategie für die Reduzierung des Arbeitszeitbedarfs im steilen Gelände eine Verminderung des Einsatzes großer Arbeitsgruppen durch Mechanisierungsmaßnahmen sein sollte.

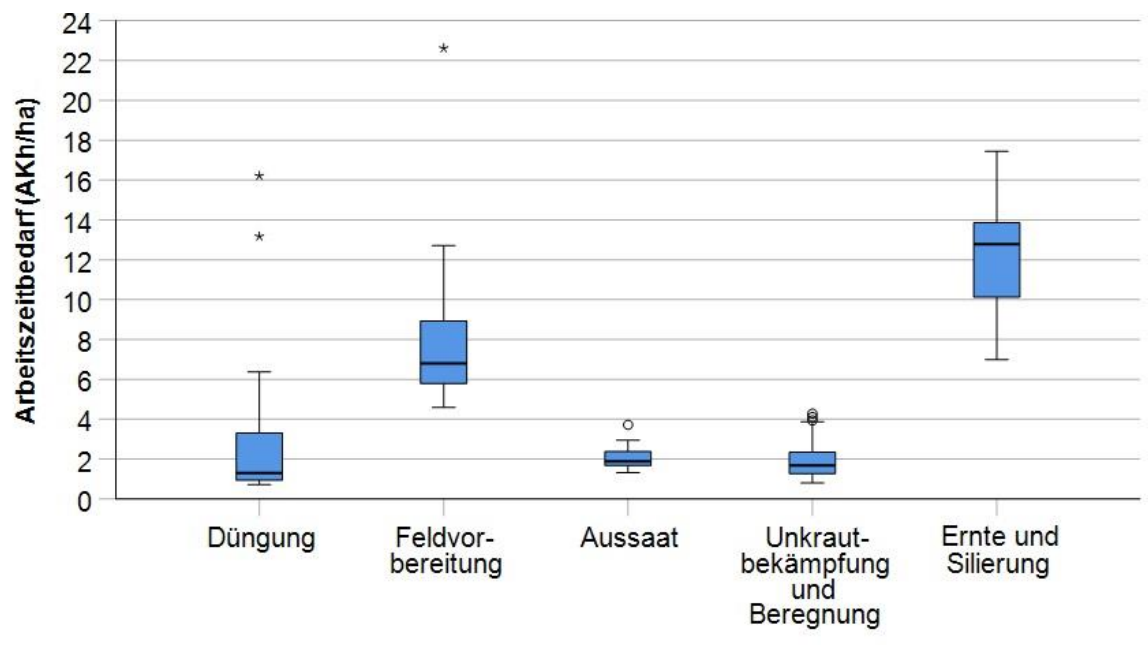

Makrokategorie

Abb. 7: Boxplot-Darstellung des Arbeitszeitbedarfs nach Makrokategorie bei Silomaisnutzung // Boxplot display of labour requirements of different macro categories for maize grown for silage. 
- Die Schlagfläche spielt dabei möglicherweise auch eine gewisse Rolle, obwohl zwei entgegengesetzte Effekte auf den Arbeitszeitbedarf zu erwarten sind: Zum einen gibt es eine negative Korrelation zwischen Schlagfläche und Arbeitszeiten, da kleine Flächen verhältnismäßig mehr Arbeit verursachen [5] [6] [10]. Zum anderen wirken sich Ungenauigkeiten (in Form von Abrundungen) bei der Erhebung der Arbeitszeiten bei sehr kleinen Flächen verhältnismäßig stärker aus. Eine Abrundung, welche eine Fehlschätzung von etwa 10 Minuten bei einer Schlagfläche von 0,2 ha bewirkt, verursacht zum Beispiel einen Fehler von 50 Minuten, wenn dieser Wert auf einen Hektar aufgerechnet wird.

- Die Mitberücksichtigung der Fahrtzeiten von der Hofstelle bis zu den bewirtschafteten Flächen ermöglicht einerseits eine realistische Quantifizierung der effektiven Arbeitszeiten des Personals unter den lokalen Bedingungen, generiert andererseits aber ein gewisses Geräusch in den Daten, weil nicht alle Schläge zeitlich gleich weit von der Hofstelle entfernt sind. Außerdem besteht eine hochsignifikante $(P=0,007)$, aber sehr schwache Korrelation nach Pearson $(R=0,192)$ zwischen der Hangneigung der Wiesenflächen und der Standardfahrtzeit (Fahrtzeit mit Ladewagen, Transporter oder Traktor). Während bei den ersten drei Hangneigungsklassen die durchschnittliche Fahrtzeit zwischen 7 und 9 Minuten beträgt (7,8 für die Klasse $<20 \%, 7,9$ für die Klasse $20-40 \%$ und 9,0 für die Klasse $40-60 \%$ ), liegt sie bei der Klasse $>60 \%$ bei etwa 14,1 Minuten. Das trägt auch zu der schnellen Zunahme des Arbeitszeitbedarfs bei zunehmender Hangneigung bei.

- Die Ergebnisse der oben genannten, herangezogenen Literatur sind unter Berücksichtigung verschiedener Einflussgrößen entstanden (Tab. 1). Für die unberücksichtigten Einflussgrößen (z.B. die Entfernung des Schlags vom Hofgebäude) werden in der Kalkulation meistens Standardwerte hinterlegt, aber diese Faktoren spielen möglicherweise eine relevante Rolle unter den Südtiroler Verhältnissen.

Unter Berücksichtigung der oben genannten Rahmenbedingungen und Einschränkungen stellen die Ergebnisse der vorliegenden Untersuchung, vor allem was die Wiesen und den Silomaisanbau anbelangt, fundierte Richtwerte für den Arbeitszeitbedarf in der landwirtschaftlichen Praxis Südtirols dar. Sie stehen somit den Beratungseinrichtungen für Berechnungen und Argumentationen zur Verfügung.

\section{DANKSAGUNGEN}

Nur dank der Begeisterung und dem Einsatz von Seiten der teilnehmenden Landwirte war es möglich, eine große Menge an genauen und zuverlässigen Daten zu sammeln. An sie richten wir unser herzlichstes Dankeschön. Wir bedanken uns bei Hermann Stuppner für die gute Zusammenarbeit und den Informationsaustausch zur partizipativen Identifizierung der Ziele der Auswertung. Ein weiteres Dankeschön geht an die Abteilung Landwirtschaft der Autonomen Provinz Bozen (Norbert Zenleser, Paul Steger, Robert Großrubatscher, Georg Tschurtschenthaler, dem Direktor der Abteilung Dr. Martin Pazeller und allen Direktoren der landwirtschaftlichen Bezirksämter), sowie dem Mitarbeiter Jan Luca Senoner der ehemaligen Dienststelle für Bergbauernberatung für die Unterstützung während der

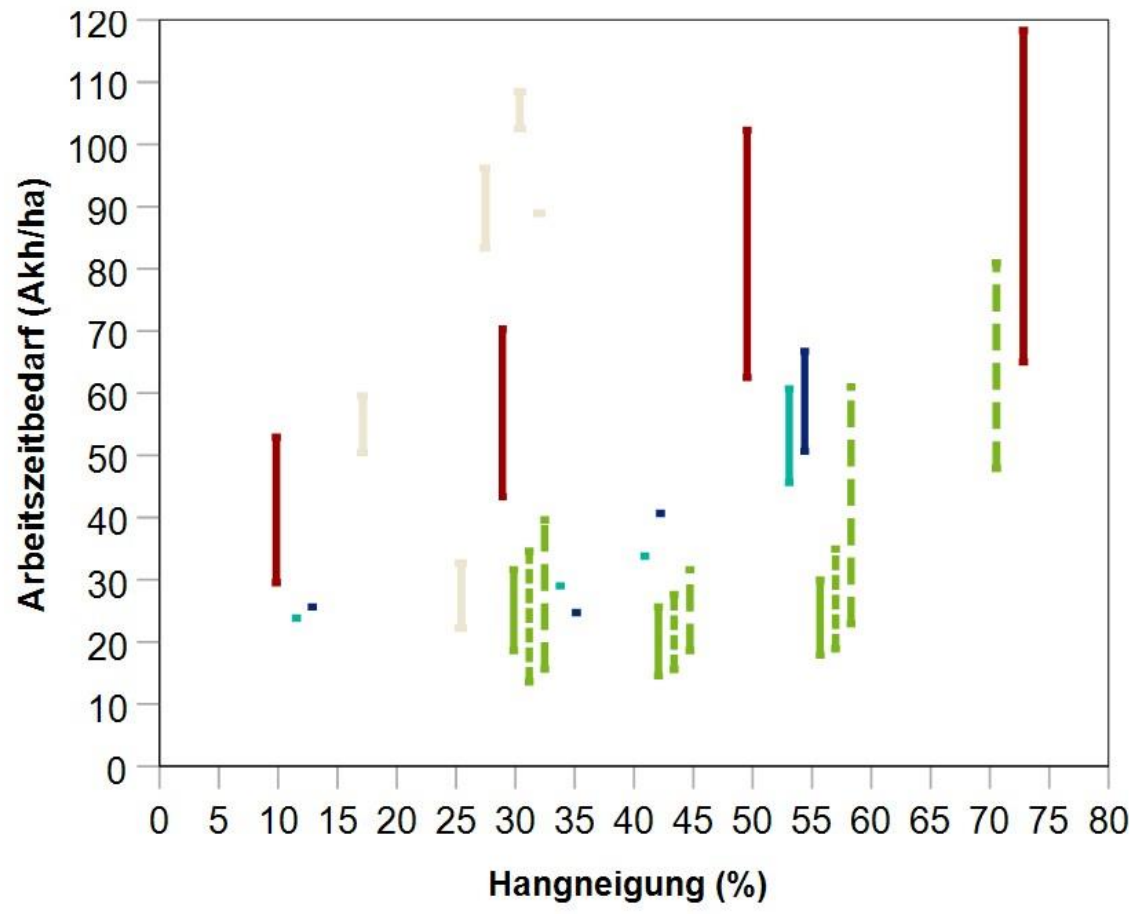

\section{Literaturquelle (Futterkonservie- rungsverfahren)}

I vorliegende Studie

I Greimel et al. 2002 (Silage)

Greimel et al. 2002 (Dürrfutter)

- BMLFUW 2008

(Silage)

7 BMLFUW 2008

(Belüftungsheu)

BMLFUW 2008

- (Bodenheu)

Schätzung nach

Schick 1995 be

Betrieben der vorliegenden Studie

Abb. 8: Grafische Gegenüberstellung des Effektes der Hangneigung auf den Gesamtarbeitszeitbedarf bei der vorliegenden Untersuchung (Auswertung mit logarithmierten Daten, Rücktransformierung des 95\%-Konfidenzintervalls) und anderen Literaturquellen. Die Balken zeigen Minimum und Maximum des Streuungsintervalls. Die Werte nach Schick (1995) beziehen sich auf einzelne Betriebe und zeigen die Streuung innerhalb der Untersuchungsperiode. // Graphical comparison of the effect of slope on the total labour requirements in the present study (analysis with logarithmtransformed data and back-transformation of the 95\% confidence interval) and other literature sources. The bars show minimum and maximum of the variation interval. The values according to Schick (1995) refer to individual farms and show the variation within the period of investigation. 
Projektzeit, das Auffinden der Betriebe und für ihre Mitarbeit bei der Datenaufzeichnung. Außerdem möchten wir uns beim Amt für Kartografie und Statistik der Autonomen Provinz Bozen für die kartografische Unterstützung und für die topografischen Eigenschaften der untersuchten Grundstücke bedanken. Martina Querini danken wir für die Mithilfe bei der Übersetzung von italienischen Texten.

Diese Arbeit wurde dank dem Aktionsplan für Forschung und Ausbildung für die Berglandwirtschaft und die Lebensmittelwissenschaften ermöglicht.

\section{ZUSAMMENFASSUNG}

Die Futterproduktion im Berggebiet ist eine wichtige Produktionsgrundlage für die viehhaltenden Betriebe sowie eine Quelle von Ökosystemdienstleistungen für die Gesellschaft, wobei die Arbeitszeiten zum Teil erheblich sind. Die Richtwerte aus anderen Ländern des Alpenraums unterliegen anderen betrieblichen und topografischen Gegebenheiten. Das Ziel dieser Untersuchung besteht deshalb darin, zuverlässige, lokale und spezifische Richtwerte für den Arbeitszeitbedarf bei der Futterproduktion im Berggebiet zu generieren. 20 Betriebe im Raum Pustertal wurden ausgewählt und 100-109 dazugehörende Schläge der Kategorien Wiese, Mähweide und Mais pro Jahr untersucht. Die Datenerhebung erfolgte von 2011 bis 2013 und die Landwirte erhoben systematisch ihre einzelnen Arbeitsschritte sowie die dazugehörenden Arbeitszeiten inkl. Auf- und Abrüstzeiten sowie Anfahrtszeiten. Die Hangneigung hat bei den Wiesen einen hochsignifikanten Effekt auf den Gesamtarbeitsbedarf, was insbesondere auch auf die starke Zunahme der Handarbeit bei der Futterernte zurückgeführt werden kann. Der Arbeitszeitbedarf bei der Futterernte war bei der höchsten Neigungsklasse um das Fünffache höher als bei der niedrigsten. Die Meereshöhe war hingegen statistisch nicht signifikant. Auch bei den Mähweiden beeinflusste nur die Hangneigung den Gesamtarbeitszeitbedarf. Beim Silomais waren Ernte und Silierung die zeitaufwändigsten Kategorien, gefolgt von der Feldvorbereitung. Unter Berücksichtigung der gegebenen Rahmenbedingungen und Einschränkungen liefern die Ergebnisse brauchbare Richtwerte hinsichtlich des Arbeitszeitbedarfs in der landwirtschaftlichen Praxis Südtirols.

\section{RIASSUNTO}

La produzione di foraggio nelle aree montane è un'importante base produttiva per le aziende zootecniche e una fonte di servizi ecosistemici per la società. Tuttavia i tempi di lavoro sono talvolta considerevoli. I valori di riferimento di altri paesi della regione alpina sono soggetti ad altre condizioni aziendali e topografiche. Lo scopo di questo studio è quindi quello di generare valori $d$ riferimento affidabili, locali e specifici relativi al fabbisogno di manodopera per ettaro per la produzione di foraggio nelle aree di montagna. Sono state selezionate 20 aziende agricole nella zona della Val Pusteria e sono state analizzate da 100 a 109 parcelle per anno afferenti alle categorie prato, prato-pascolo e mais da trinciato. La raccolta dei dati si è svolta dal 2011 al 2013 e gli agricoltori hanno registrato sistematicamente i tempi di lavoro alle singole operazioni in campo, compresi i tempi di montaggio e smontaggio e i tempi di viaggio. La pendenza ha mostrato un effetto altamente significativo sul fabbisogno totale di manodopera per la gestione dei prati, attribuibile in particolare al forte aumento del lavoro manuale per la raccolta del foraggio, con un fabbisogno di manodopera per la raccolta del foraggio della classe di pendenza più elevata che era circa il quintuplo di quello della classe di pendenza più bassa. L'altitudine, invece, non ha mostrato effetti statisticamente significativi. Anche per i prati-pascolo solo la pendenza ha influenzato il fabbisogno totale di manodopera. La raccolta e l'insilamento si sono dimostrate le categorie di lavoro più onerose per il mais da trinciato, seguite dalle lavorazioni del terreno. Tenendo conto della metodologia per il rilevamento dei dati e dei relativi vincoli, i risultati forniscono utili valori di riferimento per il fabbisogno di manodopera nella pratica agricola dell'Alto Adige. 


\section{LITERATUR}

[1] Peratoner G., Kasal A., Plitzner C. (2010). Stima del bilancio foraggero per l'Alto Adige. Quaderno SoZooAlp 6, 111-122.

[2] Pötsch E. (2010). Multifunktionalität und Bewirtschaftungsvielfalt im österreichischen Grünland. In: Lehr- und Forschungszentrum Raumberg-Gumpenstein (ed.). Biodiversität im Grünland. 16. Alpenländisches Expertenforum, Raumberg-Gumpenstein, Österreich March 11, 2010. Lehr- und Forschungszentrum für Landwirtschaft Raumberg-Gumpenstein, Irdning, Österreich, pp. 1-10.

[3] Niedrist G., Tasser E., Lüth C. et al. (2009). Plant diversity declines with recent land use changes in European Alps. Plant Ecology 202:195, 195-210, DOI: 10.1007/s11258008-9487-x.

[4] Greimel M., Handler F., Blumauer E. (2002) Arbeitszeitbedarf in der österreichischen Landwirtschaft. Bundesanstalt für alpenländische Landwirtschaft Gumpenstein, Irdning, Österreich. Retrieved November 20, 2020, from https://raumberg-gumpenstein.at/component/search/?searchword=arbeitszeitbericht\&searchphrase $=$ all $\&$ Itemid $=425$.

[5] Schick M., Stark R. (2002). Arbeitswirtschaftliche Kennzahlen zur Raufutterernte. (FATBerichte ; 588). Eidgenössische Forschungsanstalt für Agrarwirtschaft und Landtechnik (FAT), Tänikon, Schweiz. Retrieved November 20, 2020, from http://link.ira.agroscope.ch/de-CH/publication/18358.

[6] Peratoner G., De Ros G., Senoner J.L. et al. (2013). Costs of forage production in disadvantaged mountain areas. Grassland Science in Europe 18, 332-334.
[7] Peratoner G., de Ros G., Senoner J.L. et al. (2017). Effect of slope and altitude on the costs of forage production in mountain areas. Grassland Science in Europe 22, 215217.

[8] Peratoner G., Figl U., Florian C. et al. (2015). Studio dei costi di produzione del foraggio nella Provincia di Bolzano (BLW-gw-11-1). Relazione finale di progetto. Land- und Forstwirtschaftliches Versuchszentrum Laimburg, Pfatten, Italien, p. 34.

[9] Autonome Provinz Bozen - Südtirol (ed.) (2008). Erschwernispunkte in der Berglandwirtschaft, Bozen. Retrieved November 20, 2020, from http://www.provinz.bz.it/landforstwirtschaft/landwirtschaft/publikationen.asp?publ action=300\&publ image id=156548.

[10] Schick M. (1995). Arbeit auf dem Bergbetrieb. (FAT-Berichte ; 472). Eidgenössische Forschungsanstalt für Agrarwirtschaft und Landtechnik (FAT), Tänikon, Schweiz. Retrieved November 20, 2020, from http://link.ira.agroscope.ch/de-CH/publication/18536.

[11] Schick M. (2001). Weidehaltung Milchvieh (FAT-Berichte ; 562). Eidgenössische Forschungsanstalt für Agrarwirtschaft und Landtechnik (FAT), Tänikon, Schweiz. Retrieved November 20, 2020, from http://link.ira.agroscope.ch/de-CH/publication/18539.

[12] Lauber S., Schick M., Schiess I. et al. (2005) Transporte im Berggebiet. Geschwindigkeit und Treibstoffverbrauch auf steilen Strassen. (FAT-Berichte ; 637). Eidgenössische Forschungsanstalt für Agrarwirtschaft und Landtechnik (FAT), Tänikon, Schweiz. Retrieved November 20, 2020, from http://link.ira.agroscope.ch/de-CH/publication/17702.
[13] Dilger M., Faulhaber I. (2006). Materialsammlung Futterwirtschaft. Daten, Fakten und Berechnungsgrundlagen zu den Kosten der Futtererzeugung und der Futterwirtschaft. (LfL-Information). Bayerische Landesanstalt für Landwirtschaft, Freising-Weihenstephan, Deutschland. Retrieved No vember 20, 2020, from https://www.Ifl.bayern.de/mam/cms07/publikationen/daten/informationen/p_22478.pdf.

[14] Bundesministerium für Land- und Forstwirtschaft, Umwelt und Wasserwirtschaft (BMLFUW) (ed.) (2008). Deckungsbeiträge und Daten für die Betriebsplanung 2008. Bundesministerium für Land- und Forstwirtschaft, Umwelt und Wasserwirtschaft, Wien Österreich. Retrieved November 19, 2020, from

https://www.agraroekonomik.at/filead$\mathrm{min} /$ download/Deckungsbeitraege und Daten 2008.pdf.

[15] Riegel M., Schick M., Hartmann W. (2009). Arbeitszeit- und Investitionsbedarf für Weideeinrichtungen. Landtechnik 64 (1), 45-47. Retrieved November 19, 2020, from https://web.ar-

chive.org/web/20180509120257id/https:// www.landtechnik-online.eu/ojs-2.4.5/index.php/landtechnik/article/viewFile/20091-045-047/1497.

[16] Hawkins D.M., Basak S.C., Mills D. (2003). Assessing model fit by cross-validation. Journal of Chemical Information and Computer Science 43, 579-586 DOI: $10.1021 / \mathrm{ci025626i.}$

[17] Kozak M., Piepho H.-P. (2018). What's normal anyway? Residual plots are more telling than significance tests when checking ANOVA assumptions. Journal of Agronomy and Crop Science 204, 86-98, DOI: 10.1111/jac.12220. 


\section{ANHANG}

Tab. a: Zusammenfassende Eigenschaften der Betriebe, die am Projekt beteiligt waren. Alle Mittelwerte sind auf Betriebsebene berechnet worden (mit Ausnahme der Hangneigung, die auf Schlagebene berechnet wurde). Auch die Silomaisflächen sind bei der Berechnung berücksichtigt worden. Die unten angeführten Werte sind Mittelwerte \pm Standardabweichung. Werte ohne Standardabweichung beziehen sich auf Betriebe, die nur an einem der drei Projektjahre beteiligt waren. // Summary of the characteristics of the investigated farms. All means have been calculated at farm level (with the exception of slope, which has been calculated at field level). The silage maize areas have also been considered in the calculation. The values are means \pm standard deviation. Values without standard deviation refer to farms that participated in only one of the three project years.

\begin{tabular}{|c|c|c|c|c|c|c|c|}
\hline $\begin{array}{l}\text { Fortlaufende } \\
\text { Betriebsnum- } \\
\text { mer }\end{array}$ & $\begin{array}{l}\text { Durchschnittli- } \\
\text { che } \\
\text { Meereshöhe } \\
\text { Mean altitude }\end{array}$ & $\begin{array}{l}\text { Durchschnittli- } \\
\text { che Hang- } \\
\text { neigung } \\
\text { Mean slope }\end{array}$ & $\begin{array}{l}\text { Durchschnittli- } \\
\text { che Schlag- } \\
\text { fläche }\end{array}$ & $\begin{array}{l}\text { Futterproduk- } \\
\text { tion durch } \\
\text { Heu }\end{array}$ & $\begin{array}{l}\text { Futterpro- } \\
\text { duktion } \\
\text { durch Grassi- } \\
\text { lage } \\
\text { Forage pro- } \\
\text { duction as } \\
\text { grass silage }\end{array}$ & $\begin{array}{l}\text { Futterproduk- } \\
\text { tion durch Silo- } \\
\text { mais } \\
\text { Forage produc- } \\
\text { tion as silage } \\
\text { maize }\end{array}$ & $\begin{array}{l}\text { Futterproduk- } \\
\text { tion auf der } \\
\text { Weide } \\
\text { Forage pro- } \\
\text { duction on } \\
\text { pastures }\end{array}$ \\
\hline & (m) & (\%) & (ha) & (\%) & (\%) & (\%) & (\%) \\
\hline 1 & $813 \pm 5$ & $5,4 \pm 2,6$ & 6,5 & 55,9 & 37,9 & & 6,2 \\
\hline 2 & $838 \pm 22$ & $12,2 \pm 8,3$ & $1,4 \pm 1,0$ & 12,2 & 27,8 & 58,7 & 1,3 \\
\hline 3 & $920 \pm 22$ & $8,9 \pm 4,3$ & $1,3 \pm 0,4$ & 25,5 & 19,7 & 54,4 & 0,5 \\
\hline 4 & $1020 \pm 198$ & $20 \pm 9,7$ & $0,5 \pm 0,1$ & 93,7 & & & 6,3 \\
\hline 5 & $1113 \pm 242$ & $12,3 \pm 11,5$ & $2,8 \pm 1,4$ & 87,1 & & & 12,9 \\
\hline 6 & $1162 \pm 79$ & $28,6 \pm 4,2$ & $1,3 \pm 0,7$ & 64 & & & 36 \\
\hline 7 & $1180 \pm 29$ & $26,7 \pm 3,5$ & $1,0 \pm 1,0$ & 97,3 & 2,7 & & \\
\hline 8 & $1193 \pm 16$ & $8,1 \pm 3,8$ & $5,8 \pm 2,5$ & 51,7 & 48,3 & & \\
\hline 9 & $1208 \pm 15$ & $10,9 \pm 8,8$ & $3,6 \pm 2,7$ & 61,1 & & & 38,9 \\
\hline 10 & $1251 \pm 16$ & $14,8 \pm 6,8$ & $1,1 \pm 0,4$ & 52,7 & 32,8 & & 14,5 \\
\hline 11 & $1321 \pm 270$ & $27,5 \pm 6,2$ & $1,1 \pm 0,5$ & 99,2 & 0,8 & & \\
\hline 12 & $1321 \pm 96$ & $16,1 \pm 8,9$ & $1,8 \pm 1,1$ & 59,6 & 26,5 & & 13,9 \\
\hline 13 & $1349 \pm 74$ & $12,4 \pm 8,2$ & $1,9 \pm 1,2$ & 67,2 & 23,2 & & 9,5 \\
\hline 14 & $1350 \pm 26$ & $34,5 \pm 1,9$ & $0,6 \pm 0,4$ & 100 & & & \\
\hline 15 & $1450 \pm 34$ & $35 \pm 4,4$ & $0,9 \pm 0,2$ & 94 & & & 6 \\
\hline 16 & $1502 \pm 30$ & $23,7 \pm 5,1$ & $1,3 \pm 0,7$ & 57,7 & 30,7 & & 11,6 \\
\hline 17 & $1659 \pm 78$ & $16,1 \pm 8,4$ & $1,7 \pm 0,7$ & 100 & & & \\
\hline 18 & $1546 \pm 93$ & $15,4 \pm 5,8$ & $1,3 \pm 0,9$ & 87,1 & & & 12,9 \\
\hline 19 & $1692 \pm 158$ & $13,1 \pm 7,6$ & $2,7 \pm 2,3$ & 64,5 & 28,6 & & 6,9 \\
\hline 20 & $1717 \pm 305$ & $18,4 \pm 9,5$ & $1,3 \pm 0,7$ & 91,8 & 1,6 & & 6,6 \\
\hline
\end{tabular}


Tab. b: Kennzeichnende Größen der Datenverteilung des Arbeitszeitbedarfs in AKh/ha (10-, 25-, 75- und 90-Perzentile sowie Median und Mittelwert der nichttransformierten Daten) für die Kategorie Wiesen $/ / 10^{\text {th }}, 25 \mathrm{th}, 75^{\text {th }}, 90^{\text {th }}$ percentiles, median and arithmetic mean of the untransformed data of labour requirements (in labour hours per hectare) for the category meadows.

\begin{tabular}{|c|c|c|c|c|c|}
\hline \multirow[t]{2}{*}{$\begin{array}{l}\text { Arbeitskategorie } \\
\text { Work category }\end{array}$} & \multirow[t]{2}{*}{$\begin{array}{l}\text { Parameter } \\
\text { Parameter }\end{array}$} & \multicolumn{4}{|c|}{$\begin{array}{l}\text { Hangneigungsklasse } \\
\text { Slope class }\end{array}$} \\
\hline & & $<20 \%$ & $20-40 \%$ & $40-60 \%$ & $>60 \%$ \\
\hline \multirow{6}{*}{ Düngung } & 10-Perzentil & 1,3 & 1,6 & 1,9 & 2,6 \\
\hline & 25-Perzentil & 1,8 & 2,7 & 3,0 & 4,8 \\
\hline & Median & 2,9 & 5,5 & 7,2 & 7,5 \\
\hline & Mittelwert & 4,3 & 6,9 & 9,0 & 7,8 \\
\hline & 75-Perzentil & 6,4 & 9,8 & 12,3 & 9,7 \\
\hline & 90-Perzentil & 9,5 & 13,5 & 21,0 & 15,9 \\
\hline \multirow{6}{*}{ Grünlandpflege } & 10-Perzentil & 0,4 & 1,0 & 1,2 & 2,7 \\
\hline & 25-Perzentil & 0,7 & 1,4 & 2,6 & 4,0 \\
\hline & Median & 1,2 & 2,7 & 5,3 & 6,2 \\
\hline & Mittelwert & 2,1 & 5,0 & 6,8 & 11,6 \\
\hline & 75-Perzentil & 2,3 & 6,2 & 9,7 & 19,7 \\
\hline & 90-Perzentil & 4,0 & 10,2 & 14,9 & 30,7 \\
\hline \multirow{6}{*}{ Handarbeit bei Futterernte } & 10-Perzentil & 1,1 & 3,2 & 10,4 & 15,7 \\
\hline & 25-Perzentil & 2,1 & 5,9 & 19,2 & 37,4 \\
\hline & Median & 5,5 & 12,9 & 38,9 & 46,8 \\
\hline & Mittelwert & 9,2 & 23,2 & 45,4 & 56,6 \\
\hline & 75-Perzentil & 13,1 & 33,1 & 56,6 & 75,5 \\
\hline & 90-Perzentil & 22,3 & 62,1 & 95,9 & 120,0 \\
\hline \multirow{6}{*}{ Mechanisierte Futterernte } & 10-Perzentil & 6,2 & 8,3 & 7,7 & 7,0 \\
\hline & 25-Perzentil & 7,8 & 9,6 & 9,7 & 12,5 \\
\hline & Median & 11,7 & 13,0 & 13,1 & 19,2 \\
\hline & Mittelwert & 12,7 & 14,0 & 14,7 & 19,4 \\
\hline & 75-Perzentil & 15,9 & 17,4 & 19,9 & 25,1 \\
\hline & 90-Perzentil & 21,2 & 21,5 & 23,8 & 29,8 \\
\hline \multirow{6}{*}{ Aufladen-Transport } & 10-Perzentil & 1,4 & 1,6 & 2,3 & 3,5 \\
\hline & 25-Perzentil & 1,7 & 2,7 & 3,3 & 5,4 \\
\hline & Median & 2,8 & 4,0 & 4,6 & 7,4 \\
\hline & Mittelwert & 3,7 & 4,5 & 5,3 & 8,1 \\
\hline & 75-Perzentil & 4,3 & 5,9 & 6,7 & 10,8 \\
\hline & 90-Perzentil & 5,3 & 7,9 & 8,4 & 13,8 \\
\hline \multirow{6}{*}{ Arbeit im Stadel } & 10-Perzentil & 0,3 & 0,3 & 0,6 & 0,5 \\
\hline & 25-Perzentil & 0,7 & 0,7 & 1,6 & 1,0 \\
\hline & Median & 1,5 & 1,5 & 2,1 & 1,4 \\
\hline & Mittelwert & 1,6 & 1,6 & 2,7 & 1,8 \\
\hline & 75-Perzentil & 2,3 & 2,3 & 3,3 & 2,2 \\
\hline & 90-Perzentil & 3,0 & 3,0 & 4,5 & 4,4 \\
\hline
\end{tabular}


Tab. b (Fortsetzung) // (continuation).

\begin{tabular}{|c|c|c|c|c|c|}
\hline \multirow[t]{2}{*}{$\begin{array}{l}\text { Arbeitskategorie } \\
\text { Work category }\end{array}$} & \multirow[t]{2}{*}{$\begin{array}{l}\text { Parameter } \\
\text { Parameter }\end{array}$} & \multicolumn{4}{|c|}{$\begin{array}{l}\text { Hangneigungsklasse } \\
\text { slope class }\end{array}$} \\
\hline & & $<20 \%$ & $20-40 \%$ & $40-60 \%$ & $>60 \%$ \\
\hline \multirow{6}{*}{ Ohne Kategoriezuordnung } & 10-Perzentil & 0,3 & 1,3 & 1,0 & 2,4 \\
\hline & 25-Perzentil & 0,5 & 2,8 & 2,0 & 7,1 \\
\hline & Median & 4,3 & 5,3 & 6,4 & 9,4 \\
\hline & Mittelwert & 3,3 & 8,7 & 8,1 & 9,2 \\
\hline & 75-Perzentil & 5,6 & 11,4 & 10,2 & 12,0 \\
\hline & 90-Perzentil & 5,9 & 25,7 & 23,8 & 17,0 \\
\hline \multirow{6}{*}{ Gesamtarbeitszeitbedarf } & 10-Perzentil & 20,5 & 26,2 & 36,8 & 42,9 \\
\hline & 25-Perzentil & 22,4 & 29,8 & 51,0 & 69,3 \\
\hline & Median & 38,6 & 51,0 & 74,5 & 95,7 \\
\hline & Mittelwert & 37,7 & 62,3 & 83,0 & 101,0 \\
\hline & 75-Perzentil & 46,4 & 72,4 & 100,9 & 128,0 \\
\hline & 90-Perzentil & 61,6 & 129,8 & 152,4 & 172,3 \\
\hline
\end{tabular}


Tab. c: Kennzeichnende Größen der Datenverteilung des Arbeitszeitbedarfs in AKh/ha (Minimum, Mittelwert \pm Standardabweichung, Median und Maximum) für die Kategorie Mähweiden // Minimum, mean \pm standard deviation, median and maximum) of the untransformed data of labour requirements (in labour hours per hectare) for grassland use combining mowing and grazing.

\begin{tabular}{|c|c|c|c|}
\hline \multirow{2}{*}{$\begin{array}{l}\text { Arbeitskategorie } \\
\text { Work category }\end{array}$} & \multirow{2}{*}{$\begin{array}{l}\text { Parameter } \\
\text { Parameter }\end{array}$} & \multicolumn{2}{|c|}{$\begin{array}{l}\text { Hangneigungsklasse } \\
\text { Slope class }\end{array}$} \\
\hline & & $0-40 \%$ & $>40 \%$ \\
\hline \multirow{4}{*}{ Weidearbeit } & Minimum & 1,8 & 8,0 \\
\hline & Mittelwert \pm SD & $2,1 \pm 0,3$ & $10,9 \pm 4,3$ \\
\hline & Median & 2,1 & 9,1 \\
\hline & Maximum & 2,4 & 21,8 \\
\hline \multirow{4}{*}{ Grünlandpflege } & Minimum & 0,6 & 2,6 \\
\hline & Mittelwert \pm SD & $0,7 \pm 0,1$ & \pm \\
\hline & Median & 0,8 & 4,5 \\
\hline & Maximum & 0,8 & 11,8 \\
\hline \multirow{4}{*}{$\begin{array}{l}\text { Handarbeit bei } \\
\text { Futterernte }\end{array}$} & Minimum & 0,6 & 4,1 \\
\hline & Mittelwert \pm SD & $1,2 \pm 0,9$ & $60 \pm 34,7$ \\
\hline & Median & 1,2 & 69,5 \\
\hline & Maximum & 1,9 & 104,1 \\
\hline \multirow{4}{*}{$\begin{array}{l}\text { Mechanisierte } \\
\text { Futterernte }\end{array}$} & Minimum & 4,4 & 3,4 \\
\hline & Mittelwert \pm SD & $4,7 \pm 0,4$ & $21,9 \pm 10,9$ \\
\hline & Median & 4,6 & 21,7 \\
\hline & Maximum & 5,1 & 38,7 \\
\hline \multirow{4}{*}{ Aufladen-Transport } & Minimum & 1,3 & 0,3 \\
\hline & Mittelwert \pm SD & $1,4 \pm 0,2$ & $7,7 \pm 4,0$ \\
\hline & Median & 1,4 & 8,1 \\
\hline & Maximum & 1,7 & 13,2 \\
\hline \multirow{4}{*}{ Arbeit im Stadel } & Minimum & 0,5 & 0,2 \\
\hline & Mittelwert \pm SD & $0,8 \pm 0,3$ & $6,21 \pm 3,5$ \\
\hline & Median & 0,7 & 6,3 \\
\hline & Maximum & 1,2 & 12,7 \\
\hline \multirow{4}{*}{$\begin{array}{l}\text { Ohne } \\
\text { Kategoriezurodnung }\end{array}$} & Minimum & 0,5 & 3,0 \\
\hline & Mittelwert \pm SD & 0,5 & $6,5 \pm 3,4$ \\
\hline & Median & 0,5 & 6,4 \\
\hline & Maximum & 0,5 & 9,9 \\
\hline
\end{tabular}


Tab. d: Kennzeichnende Größen der Datenverteilung des Arbeitszeitbedarfs in AKh/ha (10-, 25-, 75- und 90-Perzentile sowie Median und Mittelwert der nichttransformierten Daten) für die Kategorie Silomais // 10 th 25 th, $75^{\text {th }}, 90^{\text {th }}$ percentiles, median and arithmetic mean of the untransformed data of labour requirements (in labour hours per hectare) for silage maize.

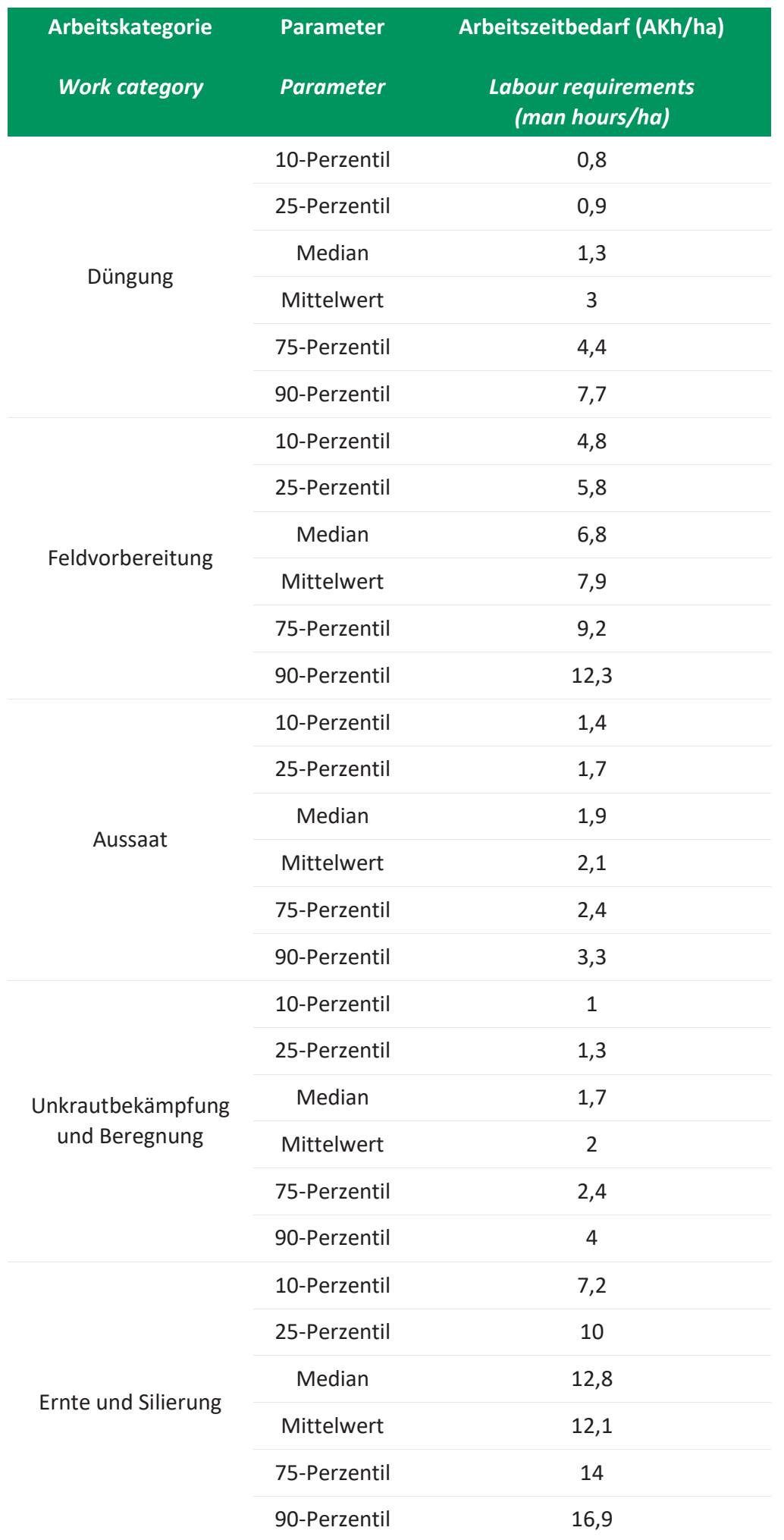




\section{(c) (i) (8)}

Dieses Werk ist lizenziert unter einer Creative Commons Namensnennung-Nicht kommerziell 4.0 International Lizenz. Quest'opera è distribuita con Licenza Creative Commons Attribuzione - Non commerciale 4.0 Internazionale.

This work is licensed under a Creative Commons Attribution-NonCommercial 4.0 International License.

Für alle Abbildungen und Tabellen ohne Nennung des Urhebers gilt: @ Versuchszentrum Laimburg. Per tutte le immagini e tabelle senza menzione dell'artefice vale: (c) Centro di Sperimentazione Laimburg. For all figures and tables without mention of the originator applies: (C) Laimburg Research Centre. 